\title{
Diversidad industrial en las provincias argentinas y factores económicos asociados (1996-2012)
}

\author{
Andrea Belmartino \\ Tesis de Maestría \\ Maestría en Economía \\ Universidad Nacional de La Plata
}

Directora: Dra. Daniela Calá (UNMdP)

Co- director: Dr. Javier Alejo (UNLP)

Noviembre, 2016 


\title{
Diversidad industrial en las provincias argentinas y factores económicos asociados (1996-2012)
}

\author{
Andrea Belmartino *
}

\begin{abstract}
Resumen
El objetivo de esta tesis es estudiar la diversidad productiva de la industria argentina en las veinticuatro jurisdicciones en las que se divide el país, y examinar los factores económicos regionales asociados a dicho fenómeno, a lo largo del período 19962012. Para ello, se estima un modelo econométrico con efectos fijos por provincias. Los resultados indican que la diversidad industrial de las provincias se asocia positivamente con el grado de desarrollo, el tamaño de la región, una mayor urbanización y las capacidades territoriales. Por otra parte, la diversidad industrial se asocia negativamente a la salida de empresas.
\end{abstract}

Clasificación JEL: L60, R11

Palabras clave:diversidad productiva, industria manufacturera argentina, ciencia regional

\begin{abstract}
The aim of this thesis is to analyze the regional productive diversity in Argentina and its associated factors (1996-2012). We estimate a panel data model with fixed effects at provincial level. The results show that regional diversity is positively related to the degree of development, region size, greater urbanization and territorial capabilities. Finally, diversity is negative related with firms exit.
\end{abstract}

JEL classification: L60, R11

Key words: productive diversity, argentine manufacture industry, regional science

\footnotetext{
* Centro de Investigaciones Económicas (Grupo: Análisis Industrial) - Facultad de Ciencias Económicas y Sociales (FCEyS)- Universidad Nacional de Mar del Plata (UNMdP). Contacto: belmartino@mdp.edu.ar. * Agradezco muy especialmente a Daniela Calá y a Javier Alejo por su valioso apoyo y guía. También al Observatorio de Empleo y Dinámica Empresarial (Ministerio de Trabajo, Empleo y Seguridad Social de la Nación) por facilitar el acceso a la base de datos. A Natacha Liseras, Fernando Graña, Miriam Berges, a la Secretaría de Investigación y al Centro de Documentación de la FCEyS-UNMdP, por el apoyo brindado. Por las sugerencias y comentarios a José Borello, a los integrantes del Grupo de Análisis Industrial, a Lucía Echeverría, a los participantes de la Red Pymes Mercosur y a los participantes seminario de tesis de Maestría. Finalmente, a mis padres y a Lautaro Rotta por su confianza y apoyo. Los errores son de mi exclusiva responsabilidad.
} 


\section{Índice}

1 Introducción $\quad 3$

2 Marco de referencia $\quad 4$

2.1 Nueva Geografía Económica . . . . . . . . . . . . . . . . . . . . . . . . . . . 4

2.2 Recursos y capacidades regionales . . . . . . . . . . . . . . . 5

3 Antecedentes empíricos $\quad 6$

4 Metodología $\quad 8$

4.1 Fuentes de información . . . . . . . . . . . . . . . . 8

4.2 Índice de diversidad productiva . . . . . . . . . . . . . . . . 9

4.3 Modelo a estimar . . . . . . . . . . . . . . . . . . 9

5 Sector industrial en Argentina $\quad 11$

6 Resultados $\quad \mathbf{1 3}$

6.1 Diversidad productiva de la industria a nivel nacional y regional (1996 - 2012) 13

6.2 Factores económicos asociados a la diversidad productiva regional . . . . . . 18

7 Análisis de robustez $\quad 21$

8 Reflexiones finales $\quad 24$

$\begin{array}{ll}\text { Referencias } & \mathbf{2 7}\end{array}$

Anexo I. Clasificación Industrial Internacional Uniforme 31

Anexo II. Evolución del índice de diversidad productiva por provincias $\quad 32$

Anexo III. Evolución del empleo industrial por rama de actividad 35 


\section{Introducción}

La diversidad productiva es un elemento clave en el diseño de estrategias de desarrollo de los países. La variedad de bienes producidos y exportados está relacionada con el desarrollo económico en sus etapas iniciales (Imbs y Wacziarg, 2003; Klinger y Lederman, 2004). A su vez, existen beneficios adicionales asociados a estructuras productivas diversas, tales como la promoción de la innovación y la transmisión de conocimiento (Duranton y Puga, 2000; Frenken et al., 2007), la reducción de la vulnerabilidad a shocks externos (Ghosh y Ostry, 1994; Kosacoff y Ramos, 1999; Haddad et al., 2010), incentivos para el ingreso de nuevas empresas (Guesnier, 1994; Reynolds et al., 1994) y mayores oportunidades de inversión (Al-Marhubi, 2000; Herzer y Nowak-Lehnmann, 2006; Hesse, 2009).

En este marco, la diversidad juega un papel central en el diseño de políticas industriales de desarrollo local. En este sentido, es importante conocer cuán diversas son las estructuras productivas regionales y cómo han evolucionado a lo largo del tiempo. Esta tesis tiene un doble objetivo: por un lado, describir la evolución de la diversidad productiva de la industria en las provincias argentinas, entre 1996 y 2012, y por otro, examinar la relación entre la diversidad industrial y algunos factores económicos regionales asociados. A partir de ello, se pretende generar información relevante que pueda ser utilizada en el diseño de políticas de desarrollo industrial a escala regional.

Los resultados obtenidos permiten avanzar en la comprensión de la diversidad productiva en Argentina, un tema destacado en los lineamientos del Plan Estratégico Industrial 2020 (PEI 2020) (Ministerio de Industria, 2011). Este plan explicita la importancia de generar acciones que promuevan el desarrollo de una trama productiva más diversa, lo cual se considera imprescindible para lograr un crecimiento sustentable y con equidad (Porta, 2016). Del mismo modo, desde la Comisión de Economía para América Latina (CEPAL) se señala a la diversidad productiva como un mecanismo para desarrollar nuevas capacidades tecnológicas en el marco de la implementación de políticas industriales selectivas (Lavarello y Sarabia, 2015). Por el contrario, los trabajos académicos que estudian la diversidad productiva en Argentina son aún escasos y se enfocan fundamentalmente en el análisis de las exportaciones a nivel país (Bebczuk y Berrettoni, 2006), o en la distribución del empleo 
de determinadas áreas geográficas (Mazorra y Beccaria, 2007; Rojo Brizuela y Rotondo, 2006).

La tesis se estructura de la siguiente forma: en primer lugar, se sintetizan los principales aportes teóricos y empíricos que permiten identificar y comprender los factores económicos asociados a la diversidad productiva regional. Luego, se describen la fuente de datos empleada, el índice de diversidad utilizado y la estrategia de estimación seleccionada. A continuación, se presentan los resultados y, finalmente, algunas reflexiones y posibles extensiones.

\section{Marco de referencia}

Este trabajo se enmarca en el campo de la Ciencia Regional (Regional Science), es decir, en el conjunto de estudios que incorporan la dimensión territorial y sus heterogeneidades en el análisis económico, permitiendo avanzar más allá de los supuestos neoclásicos (Moncayo Jiménez, 2001). Si bien este campo de la ciencia ha proporcionado modelos teóricos que permiten el estudio riguroso de ciertos temas como el crecimiento regional o la concentración territorial de la actividad económica (Capello, 2006) aún no existen modelos que describan adecuadamente el fenómeno de la diversidad productiva regional. Es por eso que los estudios empíricos sobre el tema (ver sección 3) suelen utilizar especificaciones econométricas derivadas ad hoc, contemplando la inclusión de elementos procedentes de distintos marcos teóricos. En particular, esta tesis toma argumentos provenientes de dos enfoques: a) Nueva Geografía Económica (NGE)(Krugman 1991; 1995; 1998; 1999) y b) Recursos y Capacidades (Penrose, 1959; Teece et al., 1980; Montgomery, 1994).

\subsection{Nueva Geografía Económica}

Este enfoque se compone de un conjunto de modelos de equilibrio general en una estructura de competencia imperfecta, que contemplan la existencia de rendimientos crecientes a escala, costos de transporte y la posibilidad de desplazamiento territorial de los factores productivos y de los consumidores (Krugman, 1991; Fujita y Krugman, 2004). Si bien estos modelos generan una gran variedad de resultados dependiendo de los supuestos específicos, 
la NGE resalta una serie de elementos que pueden explicar la diversidad productiva en las grandes aglomeraciones (Bishop y Gripaios, 2007).

Krugman (1998) plantea la existencia de dos fuerzas contrapuestas, las centrípetas (que concentran la actividad económica) y las centrífugas (que la disipan) ${ }^{1}$. Las primeras tienen lugar producto de una causalidad circular, por un lado, los trabajadores encuentran en los grandes centros urbanos infraestructuras de mejor calidad, salarios reales más elevados y una mayor variedad de bienes. Por otro, las empresas prefieren localizarse en grandes mercados para explotar las economías de escala internas y externas.

Por su parte, al interior del concepto de economías externas se suele distinguir entre las de localización y las de urbanización. Las primeras se refieren a los beneficios asociados a la proximidad de empresas del mismo sector industrial (mercado de trabajo especializado, proveedores, etc.), mientras que las segundas son independientes del sector industrial (servicios públicos, financieros, comerciales, transferencia de conocimiento) (Hoover, 1936).

Finalmente, si bien no significan exactamente lo mismo, el término economías de aglomeración es empleado como sinónimo de economías externas (Aláez Aller et al., 2001). En este sentido, desde una perspectiva dinámica, las economías de aglomeración de Jacobs² implican que un entorno más urbanizado, proclive a la transmisión de conocimientos e innovación, será más propicio para la diversidad productiva (Glaeser et al., 1992; Mc Cann y van Oort, 2009).

\subsection{Recursos y capacidades regionales}

El enfoque "basado en los recursos" (Resource-based view) surge a partir del aporte de Penrose (1959) al análisis de las firmas. Bajo esta óptica, una empresa es analizada en base a la dotación de recursos que la componen (Teece et al., 1980; Montgomery, 1994). Esta teoría también es aplicada a las regiones, que pueden ser definidas por su dotación de recursos tangibles (como la infraestructura) e intangibles (como el capital

\footnotetext{
${ }^{1}$ Las fuerzas centrífugas se originan en la inmovilidad de actividades relacionadas a los recursos naturales, deseconomías externas de aglomeración (efectos de congestión) y el mayor costo de la tierra y de la mano de obra (Krugman, 1998).

${ }^{2}$ Una revisión de esta teoría y la comparación con las externalidades de Marshall-Arrow-Romer (MAR) pueden encontrarse en Glaeser et al., (1992); Feldman y Audretsh (1999); Beaudry y Schiffauerova (2009); Mc Cann y van Oort (2009); Marra (2011); entre otros.
} 
humano o la calidad de sus instituciones) (Lawson, 1999; Boschma, 2004; Neffke et al., 2014; Boschma, 2015). Dado que la dotación de recursos o capacidades condicionan las actividades productivas que pueden llevar a cabo las firmas de una región, es necesario incluirlos como un factor asociado al grado y a la evolución de la diversidad productiva regional.

En esta línea, un enfoque más moderno propuesto por Hidalgo et al., (2007), analiza la red de relaciones entre productos y/o industrias, originadas en vínculos de insumoproducto, complementariedades tecnológicas y utilización de similares capacidades o infraestructuras. De acuerdo a este enfoque, el desarrollo de mayores capacidades, entendidas éstas en un sentido amplio (productivas, organizativas, institucionales, etc.) permite ampliar el abanico productivo e incluso producir bienes de mayor complejidad. Es por eso que los países tienden a diversificarse hacia productos cercanos a los que ya exportan, es decir, bienes que requieren capacidades similares. A nivel regional, el proceso de diversidad también tiende a ocurrir en actividades relacionadas a las existentes (Neffke et al., 2011), y se ve afectado por la historia industrial local, las capacidades acumuladas en el territorio y el sendero previo (path-dependence) transitado por la región (Martin y Sunley, 2006; Neffke et al., 2014).

\section{Antecedentes empíricos}

El estudio de la diversidad productiva ha sido abarcado por múltiples disciplinas y paradigmas (Ramanujan y Varadarajan, 1989). En este sentido, existen al menos tres dimensiones de análisis: firma, región y país. En particular, los estudios empíricos a nivel regional han experimentado un notable crecimiento en los últimos años (Frenken et al., 2004), especialmente en los países desarrollados ${ }^{3}$ (por ejemplo, Beckstead y Brown (2007) en Canadá; Rodgers (1957); Monastiriotis (2000) y Essletzbichler (2007) en EE.UU.; Boschma et al. (2013) en España; Bishop y Gripaios (2007) en Gran Bretaña y Boschma e Ianmarino (2007) en Italia). Los estudios académicos que siguen esta línea señalan que la diversidad

\footnotetext{
${ }^{3}$ El número de investigaciones empíricas en países en desarrollo es reducido, debido a la calidad y disponibilidad de los datos (Nachum, 2004; Hammouda et al., 2006). Aún así, estos últimos autores analizan empíricamente la diversidad productiva regional en África. Otras aplicaciones a nivel firma se encuentran para Taiwan (Aw y Batra, 1998) y Brasil, India, Chile, Argentina, entre otros (Nachum, 2004).
} 
productiva de una región está positivamente relacionada con su tamaño (Duranton y Puga, 2000; Monastiriotis, 2000; Beckstead y Brown, 2007; Bishop y Gripaios, 2007) y el grado de urbanización (Dewhurst y Mc Cann, 2003; Bishop y Gripaios, 2007). Es decir, como resultado de los elementos señalados por la NGE, los núcleos urbanos de mayor tamaño y con mayor densidad poblacional son también los más diversos.

Por otro lado, los trabajos empíricos relacionados con el enfoque de las capacidades y los recursos se enfrentan con la dificultad de identificarlos y cuantificarlos. Por ello, estas contribuciones aproximan las capacidades regionales a partir de la incorporación de medidas tales como la intensidad en investigación y desarrollo (I\&D) (Aw y Batra, 1998, Paterka y Tamberi, 2011), proporción de gerentes o responsables técnicos (Baldwin et al., 2000), empleados en ciencia y técnica (Bishop y Gripaios, 2007, Baldwin et al., 2000) o el nivel educativo de la población (Paterka y Tamberi, 2011).

Adicionalmente, algunos estudios empíricos vinculan el grado de diversidad de una región o país con su nivel de desarrollo, medido en términos del producto bruto per cápita. En esta línea, De Benedictis et al. (2009) y Paterka y Tamberi (2011) encuentran que las regiones con un menor nivel de producto per cápita poseen un menor grado de heterogeneidad productiva relativa. Al respecto, Imbs y Wacziarg (2003) demuestran que la relación entre el producto per cápita y la diversidad a nivel de países es no lineal, es decir, hasta que los países alcanzan determinados niveles de ingreso, el crecimiento se asocia positivamente a una estructura productiva y exportadora más diversa, pero luego la relación se revierte (comienzan a especializarse).

Un último grupo de trabajos explica los cambios en el grado de diversidad productiva regional a partir de elementos relacionados con la dinámica empresarial. En este sentido, identifican que un aumento de la diversidad regional puede manifestarse a partir de diferentes fenómenos:

- la creación de nuevas empresas en ramas de actividad existentes pero con menor participación relativa en el empleo regional (ramas "no tradicionales") o el crecimiento de las empresas existentes en esas ramas (Neffke et al., 2011);

- la salida de empresas o la contracción del empleo en ramas con mayor participación 
relativa en el total de ocupados de la región (ramas "tradicionales");

- el ingreso de nuevas ramas de actividad productiva (Kamien y Schwartz, 1975).

En síntesis, a partir de la revisión de la literatura se espera que la diversidad productiva regional se asocie positivamente con el grado de desarrollo regional, el tamaño de la región, una mayor urbanización y el nivel de capacidades existentes en el territorio. Asimismo, se espera que la diversidad productiva se asocie con algunas variables de dinámica empresarial, pero no es posible establecer a priori el sentido de dicha relación.

\section{Metodología}

\subsection{Fuentes de información}

La principal fuente de información es la Base de Datos para el Análisis Dinámico del Empleo (BADE), elaborada por el Observatorio de Empleo y Dinámica Empresarial (OEDE), que depende del Ministerio de Trabajo, Empleo y Seguridad Social de la Nación (MTEySS). La unidad de análisis es la empresa, la cual es identificada por su Código Único de Identificación Tributaria (CUIT). La base se construye a partir de las declaraciones de las empresas al Sistema Integrado de Jubilaciones y Pensiones, que contiene datos del empleo registrado por el sector privado en Argentina (Castillo et al., 2004). Se trabaja con datos de empleo industrial desagregados a nivel sectorial a 2 dígitos de la Clasificación Industrial Internacional Uniforme (CIIU) (ver anexo I) y a nivel regional en las 24 jurisdicciones que componen el territorio argentino (23 provincias y Ciudad Autónoma de Buenos Aires (CABA)).

Adicionalmente, se consultaron diversas fuentes de datos: Censo Nacional de Población y Vivienda, anuarios estadísticos del Instituto Nacional de Estadísticas y Censos (INDEC), direcciones de estadísticas provinciales, datos publicados por el Ministerio de Industria y el Ministerio de Ciencia y Tecnología y la misma BADE. 


\section{2 Índice de diversidad productiva}

El empleo de índices de diversidad regional permite sintetizar gran cantidad de información e interpretarla fácilmente (Mack, 2007). Los índices de diversidad productiva pueden ser calculados a partir de datos de producto o de empleo. Como es usual en la literatura, en este trabajo se emplea como medida de diversidad la inversa del índice de Herfindahl-Hirschman $(\mathrm{HH})$, construida a partir de datos del empleo regional registrado en la industria manufacturera. Dicho indicador se define como la sumatoria de la participación de cada rama en el empleo regional, elevada al cuadrado (Duranton y Puga, 2000).

$$
D P_{i}=\frac{1}{H H}=\frac{1}{\sum_{j=1}^{J}\left(\frac{E_{i}^{j}}{E_{i}}\right)^{2}}
$$

donde $E_{i}^{j}$ es el número de empleados en la rama j y en la región i y, $E_{i}$ es el número total de empleados industriales en la región.

\subsection{Modelo a estimar}

A fin de examinar la relación entre la diversidad productiva de la industria y los factores económicos asociados se estima un modelo econométrico de datos de panel. Una de las principales ventajas de su utilización consiste en el control de heterogeneidad tanto entre individuos como en el tiempo (Baltagi, 2001). Así, es posible controlar algunas características de las provincias (observables o no) que no cambian mucho en el tiempo (por ejemplo, la dotación de recursos naturales y el entorno institucional), como características temporales (ciclos, shocks, crisis).

La especificación del modelo se presenta en la ecuación (2), en donde se incluyen efectos fijos por provincia y dummies por año para captar efectos temporales $\left(\theta_{t}\right)$. La variable dependiente $\left(D P_{i t}\right)$ es el índice de diversidad definido en la ecuación (1).

$$
\begin{aligned}
D P_{i} t & =\beta_{0}+\beta_{1} \text { PBGpc }_{i t-1}+\beta_{2} \text { Densidad }_{i t-1}+\beta_{3}{\text { GastoI } \& D_{i t-1}} \\
& +\beta_{4} \text { P-expo }_{i t-1}+\beta_{5} \text { Entrada }_{i t-1}+\beta_{6} \text { Salida }_{i t-1}+\beta_{7} \theta_{t}+\varepsilon_{i t}
\end{aligned}
$$


donde los subíndices se refieren a la región $i$ y al tiempo $t$. A continuación, se describen las variables explicativas del modelo, su signo esperado y la fuente de datos. Dicha información es sintetizada en la tabla 1.

En primer lugar, se espera que el grado de desarrollo, operacionalizado a partir del Producto Bruto Geográfico per cápita (PBGpc) se relacione positivamente con su diversidad productiva (De Benedictis et al., 2009, Paterka y Tamberi, 2011). Se trabaja con datos de PBG provincial publicados por el Centro de Estudios para la Producción (Ministerio de Industria de la Nación) y por las direcciones de estadísticas provinciales. Los datos son deflactados con el índice de precios implícitos (IPI) de Argentina ${ }^{4}$, dado que no existen datos de PBGpc a precios constantes (ni índices de precios) para todas las provincias a lo largo del período analizado.

Adicionalmente, a los efectos de evaluar la relación entre el grado de diversidad y la urbanización de cada región se incorpora la variable Densidad, medida como el cociente entre la población de cada provincia y su superficie en $\mathrm{km}^{2}$. Como medida de las capacidades regionales, se incluye, por un lado, el gasto público en investigación y desarrollo (Gasto en I\&D) y, por otro, el porcentaje de las exportaciones provinciales en el total del país (P-expo). Siguiendo a Filipescu et al., (2013) y Castellacci (2007) se entiende que la inserción en el mercado externo puede ser consecuencia del desarrollo de capacidades productivas, organizacionales o institucionales. Así, una región orientada al mercado internacional requiere de un mayor aprendizaje y sofisticación tanto de sus empresas exportadoras como de sus proveedores locales, para que se ajusten a las características de la nueva demanda. El estímulo de la demanda externa también puede incentivar el desarrollo de nuevos productos y procesos, impulsando la diversidad a través de un "tirón de la demanda" (demand-pull) (Crépon et al., 1998; Schmookler, 1966).

\footnotetext{
${ }^{4}$ Alternativamente son deflactados con el índice de precios implícitos de Buenos Aires. Los resultados se mantienen (sección: análisis de robustez).
} 
Tabla 1 | Factores asociados a la diversidad productiva regional

\begin{tabular}{|c|c|c|c|c|c|c|c|}
\hline \multirow[b]{2}{*}{ Factor asociado } & \multirow[b]{2}{*}{ Variable } & & \multirow[b]{2}{*}{ Fuente de datos } & \multicolumn{4}{|c|}{ Estadísticas descriptivas } \\
\hline & & & & Media & Desvío & Mínimo & Máximo \\
\hline Desarrollo & $P B G p c$ & + & $\begin{array}{c}\text { CEP } \\
\text { EP }\end{array}$ & 0,007 & 0,005 & 0,002 & 0,034 \\
\hline Grado de urbanización & Densidad & + & INDEC & 0,581 & 1,843 & 0,015 & 11,888 \\
\hline & Gasto_I\&D & + & INDEC & 10,535 & 1,504 & 6,968 & 15,481 \\
\hline Recursos y capacidades & $P-\operatorname{expo~}$ & + & INDEC & 3,978 & 8,01 & 0,036 & 39,447 \\
\hline & Entrada & $+/-$ & OEDE & 199,39 & 415,62 & 2,000 & 2946 \\
\hline Dinámica empresarial & Salida & $+/-$ & OEDE & 180,67 & 392,21 & 4,000 & 2540 \\
\hline
\end{tabular}

Fuente: elaboración propia. CEP: Centro de Estudios para la Producción; EP: direcciones de estadísticas provinciales; INDEC; Instituto de Estadísticas y Censos de la República Argentina; OEDE: Observatorio de Empleo y Dinámica Industrial.

\section{Sector industrial en Argentina}

El sector industrial en Argentina representa el $67 \%$ de las exportaciones (33\% de origen agropecuario y $34 \%$ de origen industrial), el $22,5 \%$ del PBI y el $20 \%$ del empleo total registrado (Fuente: BADE e INDEC). Entre 1996-2012, la participación del empleo industrial total tanto a nivel Nacional como provincial ha disminuido (tabla 2), a excepción de Tierra del Fuego (crece del 28,4\% al 35,4\%). Las provincias pertenecientes al núcleo productivo del país (Buenos Aires, Santa Fe y Córdoba) superan el promedio nacional, al igual que algunas de las provincias beneficiadas con regímenes de promoción industrial (Tierra del Fuego, San Luis, La Rioja y Catamarca). 
Tabla 2 | Porcentaje de empleo industrial

\begin{tabular}{lcc} 
& $\mathbf{1 9 9 6}$ & $\mathbf{2 0 1 2}$ \\
\hline \hline Tierra del Fuego & $28,40 \%$ & $36,90 \%$ \\
San Luis & $51,90 \%$ & $34,70 \%$ \\
La Rioja & $44,10 \%$ & $33,90 \%$ \\
Buenos Aires & $33,60 \%$ & $26,50 \%$ \\
Santa Fe & $31,00 \%$ & $46,90 \%$ \\
Jujuy & $29,80 \%$ & $22,00 \%$ \\
Catamarca & $27,10 \%$ & $21,50 \%$ \\
Misiones & $30,50 \%$ & $21,20 \%$ \\
Córdoba & $26,70 \%$ & $21,10 \%$ \\
\hline Argentina & $25,90 \%$ & $20,10 \%$ \\
\hline Mendoza & $25,30 \%$ & $19,30 \%$ \\
Entre Ríos & $21,10 \%$ & $18,80 \%$ \\
San Juan & $26,60 \%$ & $17,30 \%$ \\
Tucumán & $25,60 \%$ & $16,00 \%$ \\
Corrientes & $18,40 \%$ & $15,10 \%$ \\
Salta & $16,00 \%$ & $14,20 \%$ \\
CABA & $18,80 \%$ & $13,70 \%$ \\
Chaco & $16,90 \%$ & $12,00 \%$ \\
Chubut & $17,30 \%$ & $11,60 \%$ \\
La Pampa & $13,90 \%$ & $11,50 \%$ \\
Santiago del Estero & $15,40 \%$ & $10,70 \%$ \\
Río Negro & $10,60 \%$ & $8,90 \%$ \\
Neuquén & $11,30 \%$ & $7,90 \%$ \\
Formosa & $11,61 \%$ & $7,23 \%$ \\
Santa Cruz & $6,47 \%$ & $5,42 \%$ \\
\hline \hline Fuente: elaboración propia en base a BADE.
\end{tabular}

Un patrón que se repite a lo largo de la historia es la predominancia de la rama alimentos y bebidas dentro del sector manufacturero (Zalduendo, 1973). De hecho, en el período bajo estudio no se ha modificado de forma considerable la estructura productiva (representaba el $30 \%$ del empleo industrial registrado en 1996 y en el 2012 dicho porcentaje asciende a $28 \%$ ). Al nivel provincial, se observa que es la principal actividad industrial de la mayoría de ellas, a excepción de Chaco y Tierra del Fuego (tabla 3). En particular, en las provincias Tucumán, La Pampa, Entre Ríos, Salta, Río Negro, Mendoza y Jujuy representa más de la mitad del empleo industrial registrado. 
Tabla 3 | Participación del empleo de la principal rama industrial

\begin{tabular}{llccc}
$\begin{array}{c}\text { Rama } \\
\text { (CIIU) }\end{array}$ & Provincia & $\begin{array}{c}\text { Empleo industrial registrado (\%) } \\
\mathbf{1 9 9 6}\end{array}$ & $\mathbf{2 0 1 2}$ & $\begin{array}{c}\text { Coeficiente de variación } \\
\text { (1996- 2012) }\end{array}$ \\
\hline \hline & Argentina & $30,00 \%$ & $28,00 \%$ & 0,04 \\
& CABA & $22,37 \%$ & $15,69 \%$ & 0,13 \\
& Buenos Aires & $17,51 \%$ & $16,74 \%$ & 0,06 \\
& La Rioja & $22,16 \%$ & $28,48 \%$ & 0,1 \\
& San Luis & $20,20 \%$ & $29,78 \%$ & 0,1 \\
& Chubut & $20,80 \%$ & $30,50 \%$ & 0,1 \\
& Neuquén & $28,67 \%$ & $30,62 \%$ & 0,08 \\
& Catamarca & $25,78 \%$ & $30,85 \%$ & 0,07 \\
& Santa Fe & $37,46 \%$ & $31,24 \%$ & 0,06 \\
& Córdoba & $34,67 \%$ & $33,53 \%$ & 0,06 \\
& San Juan & $36,29 \%$ & $34,24 \%$ & 0,03 \\
$\mathbf{1 5}$ & Misiones & $38,50 \%$ & $34,98 \%$ & 0,05 \\
& Santiago del Estero $\%, 07$ \\
& Corrientes & $40,95 \%$ & $37,89 \%$ & 0,07 \\
& Santa Cruz & $48,38 \%$ & $40,65 \%$ & 0,1 \\
& Formosa & $36,86 \%$ & $44,29 \%$ & 0,11 \\
& Tucumán & $39,54 \%$ & $48,18 \%$ & 0,02 \\
\hline \hline $\mathbf{3 2}$ & La Pampa & $31,28 \%$ & $51,15 \%$ & 0,14 \\
& Entre Ríos & $60,59 \%$ & $53,55 \%$ & 0,05 \\
& Salta & $58,43 \%$ & $54,48 \%$ & 0,03 \\
& Río Negro & $61,56 \%$ & $57,88 \%$ & 0,05 \\
Mendoza & $62,84 \%$ & $61,53 \%$ & 0,03 \\
& Jujuy & $64,26 \%$ & $64,55 \%$ & 0,06 \\
\hline
\end{tabular}

Ramas 15: Elaboración de productos alimenticios y bebidas; 17: Fabricación de productos textiles y 32: Fabricación de equipo y aparatos de radio, televisión y comunicaciones. Fuente: elaboración propia en base a BADE.

\section{Resultados}

\subsection{Diversidad productiva de la industria a nivel nacional y regional $(1996-2012)$}

En los últimos veinte años, la diversidad productiva de la industria argentina se relaciona estrechamente con las fluctuaciones macroeconómicas que afectaron a la economía en general y a la industria manufacturera en particular. En la Figura 1 se observa (eje izquierdo) que la diversidad industrial es un fenómeno claramente pro-cíclico, es decir, evoluciona de forma similar al producto (eje derecho). El indicador de diversidad desciende en forma continua desde 1998 hasta llegar a un mínimo en 2002, en coincidencia con la crisis política, económica y social desencadenada en Argentina a finales de 2001. En la etapa de recuperación económica iniciada en 2003 la diversidad industrial aumenta 
en forma continua, incluso por encima de los valores previos a la crisis, alcanzando un valor máximo en 2008. A partir de ese año disminuye nuevamente, en coincidencia con la crisis financiera internacional y la apreciación gradual del tipo de cambio, sin llegar a recuperarse en períodos posteriores. La asociación positiva entre el indicador de diversidad industrial y el Producto Bruto Interno (PBIpm) es estadísticamente significativa, obteniéndose resultados análogos para la tasa de crecimiento del PBIpm (tabla 4).

Figura 1 | Evolución de la diversidad industrial en Argentina

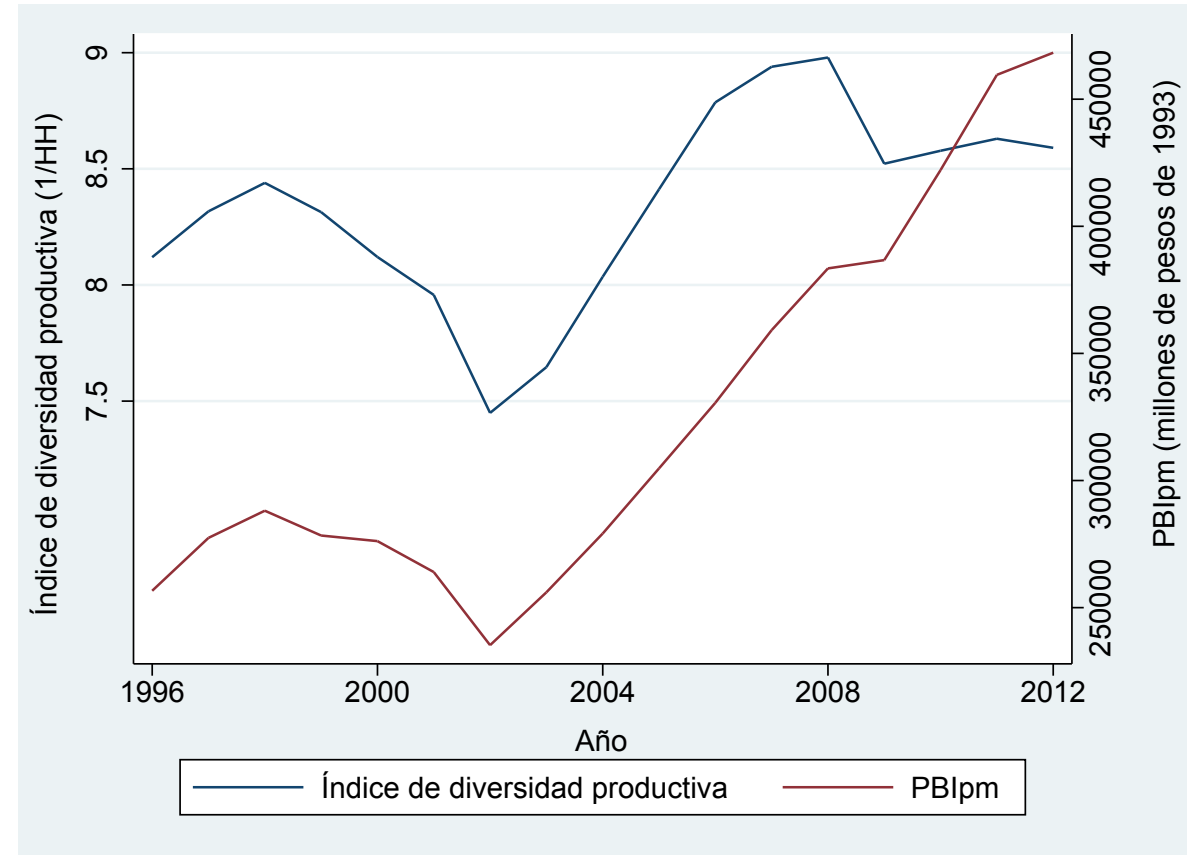

Fuente: elaboración propia en base INDEC a (PBIpm Millones de pesos de 1993) y BADE.

Tabla 4 | Correlación entre el producto y la diversidad

\begin{tabular}{cc}
\hline \hline Variables & Coeficiente de correlación de Pearson \\
\hline PBIpm / Índice de diversidad & $0,746^{*}$ \\
Tasa de crecimiento del PBIpm/ Índice de diversidad & $0,551^{* *}$ \\
\hline \hline
\end{tabular}

Fuente: elaboración propia en base INDEC a (PBIpm Millones de pesos de 1993) y BADE.

Relaciones estadísticamente significativas: ${ }^{* *} 0,05 ; * 0,10$.

Ahora bien, al interior de Argentina, ¿la diversidad también es un fenómeno procíclico en todas las regiones? ¿todas las provincias son igualmente diversas? ¿qué factores económicos se pueden asociar a un mayor o menor grado de diversidad productiva industrial? 
Lejos de ser homogéneo, el territorio argentino presenta una importante heterogeneidad estructural, que se manifiesta en una histórica desigualdad económica territorial explicada por la diversidad de recursos, la densidad poblacional, la localización industrial y la urbanización de determinadas áreas geográficas (Zalduendo, 1973; Gatto, 2007). Estas desigualdades también se observan en materia de diversidad productiva industrial (figura 2).

Figura 2 | Diversidad productiva en Argentina (2012)

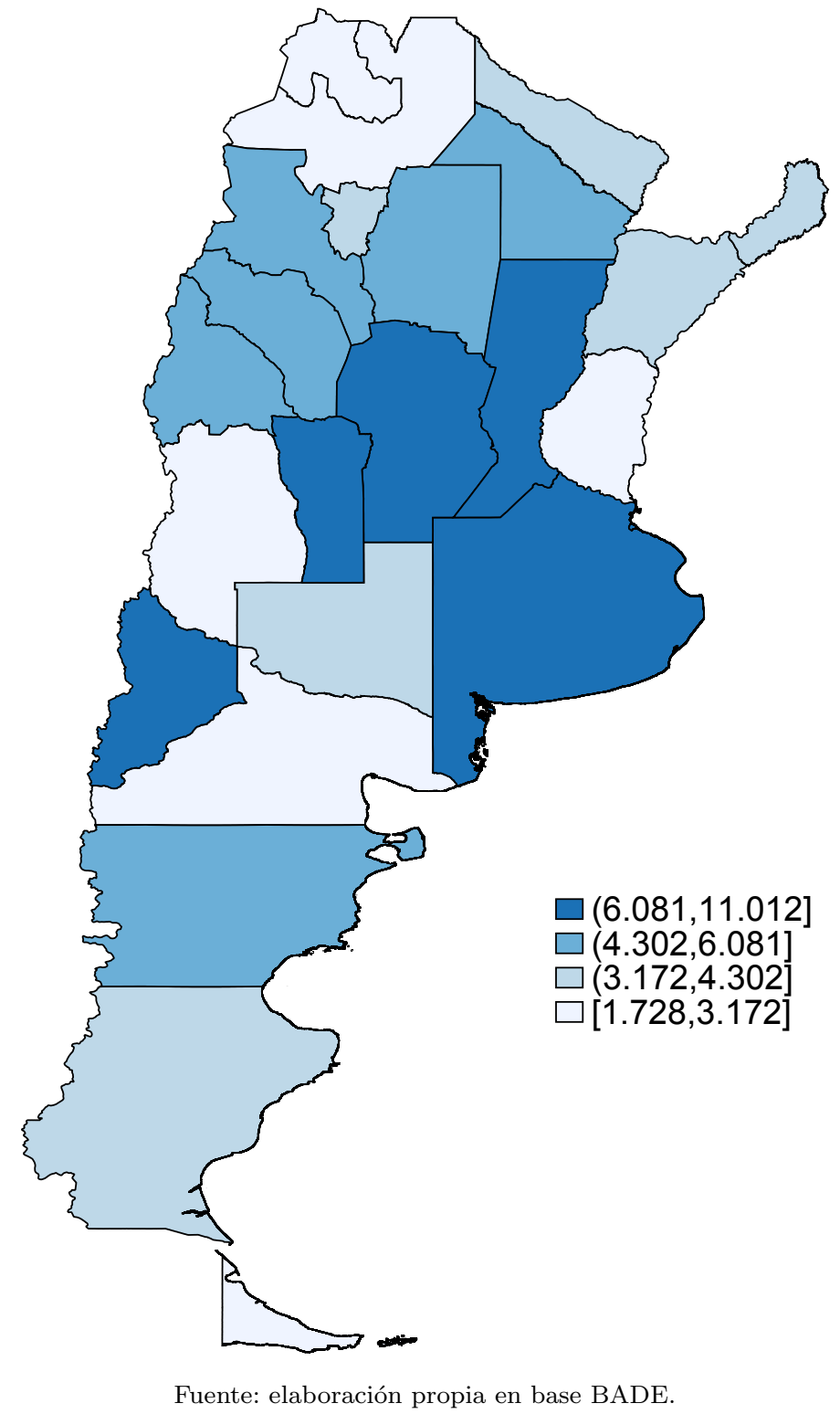

Por un lado, se distingue que las principales jurisdicciones del país (CABA, Buenos Aires, Santa Fe y Córdoba) poseen una estructura productiva más diversificada, junto 
a San Luis y Neuquén. En contraste, algunas provincias de base agroalimentaria como Salta, Jujuy, Entre Ríos, Mendoza y Río Negro, donde la elaboración de alimentos ocupa a alrededor del $60 \%$ del empleo industrial, son las menos diversas. Algo similar sucede en Tierra del Fuego, donde la industria de radio y televisión concentra el $60 \%$ del empleo registrado industrial en $2012^{5}$

En términos generales, el grado de diversidad de las provincias argentinas se ha mantenido estable en los últimos años (tabla 5). Las provincias centrales son las más diversas y, al mismo tiempo, las que presentan menos variabilidad en términos de diversidad. Se trata del núcleo productivo más fuerte del país, que concentra en 2012 el $65 \%$ de los ocupados registrados y el $70 \%$ de las exportaciones de manufacturas (fuente: BADE e INDEC).

Tabla 5 | Evolución del índice de diversidad (1996-2012)

\begin{tabular}{cllcc}
$\begin{array}{c}\text { Intensidad } \\
\text { industrial }\end{array}$ & Provincia & Índice de & Índice de & CV \\
& DP - 1996 & DP - 2012 & (1996- 2012) \\
\hline \hline \multirow{3}{*}{ Alto } & Ciudad de Buenos Aires & 9,65 & 11,01 & 0,07 \\
(centrales) & Buenos Aires & 10,29 & 10,14 & 0,03 \\
& Santa Fe & 5,73 & 6,99 & 0,06 \\
& Córdoba & 5,57 & 6,09 & 0,06 \\
\hline \multirow{3}{*}{ Alto } & San Luis & 10,45 & 7,52 & 0,09 \\
(con promoción & San Juan & 5,55 & 5,94 & 0,04 \\
industrial) & Catamarca & 6,58 & 5,41 & 0,08 \\
& La Rioja & 5,68 & 5,19 & 0,05 \\
& Tierra del Fuego & 4,33 & 2,57 & 0,15 \\
\hline & Tucumán & 3,43 & 3,44 & 0,03 \\
Medio & La Pampa & 4,68 & 3,23 & 0,17 \\
& Entre Ríos & 2,61 & 3,10 & 0,08 \\
(agro-industrial) & Salta & 2,75 & 2,77 & 0,04 \\
& Río Negro & 2,53 & 2,54 & 0,09 \\
& Mendoza & 2,45 & 2,33 & 0,06 \\
& Jujuy & 2,27 & 1,72 & 0,11 \\
\hline \multirow{3}{*}{ Bajo } & Neuquén & 6,57 & 6,58 & 0,04 \\
Chubut & 4,59 & 5,98 & 0,10 \\
(recursos no renovables) $)$ & Santa Cruz & 5,25 & 4,06 & 0,15 \\
\hline & Chaco & 4,55 & 6,06 & 0,08 \\
Bajo & Formosa & 4,85 & 4,17 & 0,13 \\
& Santiago del Estero & 4,28 & 4,43 & 0,04 \\
(complejos forestal y textil) $)$ & Corrientes & 3,74 & 4,06 & 0,08 \\
& Misiones & 3,88 & 4,00 & 0,05 \\
\hline \hline
\end{tabular}

Referencias: CV (Coeficiente de variación). Fuente: elaboración propia en base BADE.

Otro grupo de provincias relativamente diversas son las que cuentan con regímenes de promoción industrial (San Luis, San Juan, La Rioja y Catamarca). En la mayor parte

\footnotetext{
${ }^{5}$ En la tabla 4 se presenta el porcentaje de empleo que concentra la principal rama industrial en cada provincia.
} 
de ellas la diversidad ha disminuido y esto podría estar asociado al impacto decreciente de dichos regímenes sobre la estructura productiva (Donato, 2007). En el anexo II se presenta la evolución del índice por provincia.En el caso de Tierra del Fuego, se observa una disminución de la diversidad producto del aumento relativo del empleo en los sectores beneficiados por regímenes especiales (Radio y Tv.; Textil; Cuero y calzado.

Dentro de las provincias con intensidad industrial baja (en términos de empleo), se destaca Neuquén y Chubut, dónde el aumento de esa diversidad obedece al crecimiento del empleo en ramas poco tradicionales como Maquinaria y equipo o Productos de metal. En Santa Cruz la participación de Maquinaria y equipo también aumenta, pero eso no llega a impactar en el grado de diversidad, debido al importante crecimiento de la industria principal (Alimentos). Por su parte, Corrientes se registra un aumento de la participación del empleo en ramas tradicionales de la región (textil y madera), que conlleva a una disminución del índice de diversidad.

Finalmente, las provincias de base agroalimentaria son las menos diversas, como consecuencia de la elevada participación de la industria alimenticia en el total de empleo industrial regional (más del $50 \%$ ). Teniendo en cuenta el grado de desarrollo productivo de algunas provincias (como Mendoza o Entre Ríos), es posible que exista una alta diversidad al interior de la rama alimentos (diversidad relacionada), que no puede ser captada por el indicador de diversidad utilizado (inversa del índice $\mathrm{HH}$ con datos de empleo desagregados a 2 dígitos CIIU). En el anexo III se presenta la evolución de la participación del empleo industrial por rama de actividad de las provincias mencionadas.

La figura 3 muestra la evolución de la diversidad promedio en función de la agrupación provincial presentada en la tabla 3, junto con el índice a nivel nacional. Se observa que la tendencia nacional es marcada por las regiones más diversas que justamente, son las de mayor peso relativo en términos de empleo. Las demás provincias muestran una tendencia similar, a excepción de las provincias con regímenes de promoción industrial donde disminuye la diversidad en el período bajo análisis. 
Figura 3 | Evolución de la diversidad industrial por grupos de provincias

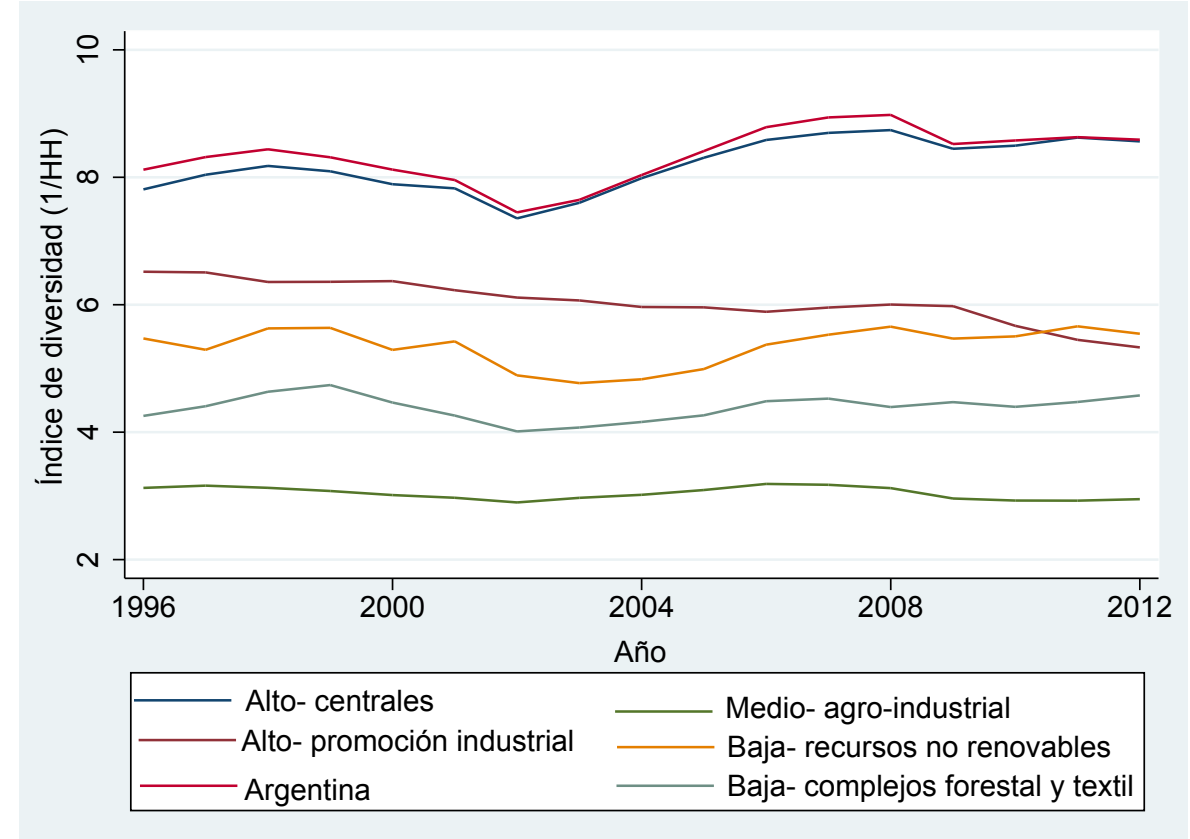

Fuente: elaboración propia en base BADE.

\subsection{Factores económicos asociados a la diversidad productiva regional}

A los efectos de identificar los factores económicos que se asocian al grado de diversidad regional se estima un modelo para datos de panel de efectos fijos (EF). Este modelo fue preferido al de efectos aleatorios debido a que se rechaza la hipótesis nula del test de Hausman $(1978)^{6}$. En la tabla 6 se presentan los resultados de la estimación, que incluyen variables dummies por año para captar el efecto temporal. Por su parte, las variables asociadas se toman rezagadas un período como estrategia ante el potencial problema de endogeneidad (Bebzuck y Berretoni, 2006).

La mayor parte de los coeficientes estimados resultan estadísticamente significativos y con el signo esperado de acuerdo con la literatura. En primer lugar, se observa la existencia de una relación directa entre el producto per cápita de la región y la diversidad productiva. Es decir, se verifica que el grado de desarrollo regional se asocia positivamente con la diversidad productiva regional ${ }^{7}$.

\footnotetext{
${ }^{6} \mathrm{El}$ supuesto clave implica que, en el modelo de efectos aleatorios no puede asumirse que las variables explicativas sean independientes del término de error, por lo cual si se rechaza la hipótesis nula, se sugiere trabajar con efectos fijos, que siempre proporciona estimaciones consistentes (Wooldridge, 2010). Las estimaciones con efectos aleatorios se encuentran disponibles para quienes las soliciten.

${ }^{7}$ En especificaciones alternativas se incluyó además el término cuadrático $\left(\mathrm{PBGpc}^{2}\right)$ para identificar si
} 
Tabla 6 | Factores asociados a la diversidad de la industria regional (Modelo 1)

\begin{tabular}{|c|c|}
\hline Factores & Modelo 1 \\
\hline PBG per cápita & $\begin{array}{c}84,8968^{*} \\
(-34,8276)\end{array}$ \\
\hline Densidad & $\begin{array}{c}0,9827^{* * *} \\
(-0,1433)\end{array}$ \\
\hline Gasto en I\&D & $\begin{array}{c}0,2095 \\
(-0,1533)\end{array}$ \\
\hline Participación en las exportaciones & $\begin{array}{l}0,0552^{*} \\
(-0,0211)\end{array}$ \\
\hline Apertura de empresas industriales & $\begin{array}{c}0,0000 \\
(-0,0002)\end{array}$ \\
\hline Cierre de empresas industriales & $\begin{array}{l}-0,0008^{*} \\
(-0,0003)\end{array}$ \\
\hline Constante & $\begin{array}{c}2,0581 \\
(-1,5958)\end{array}$ \\
\hline Test F & $57,89^{* * *}$ \\
\hline $\mathrm{R} 2(\mathrm{w})$ & 0,36 \\
\hline
\end{tabular}

Observaciones: 378 .

Sig. $* p<0,05 ; * * p<0,01 ; * * * p<0,001$

Efectos fijos por provincia, dummies por año

Variable dependiente: Índice de diversidad productiva

Variables rezagadas un período. Errores estándares robustos entre paréntesis.

Fuente: elaboración propia en base BADE.

La densidad poblacional (variable proxy del grado de urbanismo) se asocia de forma directa a la diversidad. Es decir, que se encuentra evidencia a favor de las economías de urbanización en la región. Se encuentra también una relación positiva y significativa entre la participación de cada provincia en las exportaciones nacionales y su grado de diversidad, entendiéndose que las provincias con mayor inserción en el mercado externo poseen más capacidades y una mayor historia industrial, que potencia las posibilidades de ampliar el abanico productivo de la industria de la región.

Por otro lado, si bien se esperaba que el gasto en I\&D se asocie positivamente con la diversidad, el coeficiente resulta positivo pero no significativo. Esto puede obedecer a que el gasto público en I\&D a nivel provincial pareciera no ser suficiente como para fomentar un cambio en la estructura productiva en el caso argentino.

Respecto a las variables que captan la dinámica industrial regional, se observa que la salida de empresas se asocia de forma negativa a la diversidad. Esto implicaría que

existe una relación no lineal entre diversidad regional y desarrollo, tal como la observada a nivel de países por Imbs y Wacziarg (2003). El término no resultó estadísticamente significativo. Lo cual podría deberse a las regiones argentinas son mucho más homogéneas en términos de desarrollo que los países analizados en el trabajo a nivel de países mencionado. 
las salidas se producen mayormente en las ramas menos tradicionales de cada región, ya sea por contar con menos economías de localización, escasas capacidades productivas o institucionales para mantener a esas empresas en el mercado o una infraestructura poco adecuada para su desarrollo. La entrada de empresas no resulta estadísticamente significativa, lo cual podría deberse a que se compense el efecto por ingresos en ramas tradicionales como no tradicionales o que no se pueda identificar el efecto porque en algunas provincias las entradas son escasas. En el caso de disponer de una mayor desagregación de los datos de dinámica empresarial, podría ser posible identificar efectivamente en que ramas de actividad se producen las entradas y salidas de empresas a nivel provincial.

La tabla 7 muestra los resultados de una especificación alternativa, que incluye una medida del tamaño de la región (PBG) (omitiendo la medida de grado de desarrollo (PBGpc)), a fin de analizar si, tal como sugiere la literatura, las regiones de mayor tamaño son las más diversas. Se observa que la relación entre el tamaño de la región y la diversidad es positiva y significativa, el resto de los resultados se mantienen. 
Tabla 7 | Factores asociados a la diversidad de la industria regional (Modelo 2)

\begin{tabular}{lc}
\multicolumn{1}{c}{ Factores } & Modelo 2 \\
\hline \hline PBG & $0,00001^{*}$ \\
& $(0,000)$ \\
Densidad & $0,8387^{* * *}$ \\
& $(-0,118)$ \\
Gasto en I\&D & 0,2237 \\
& $(-0,181)$ \\
Participación en las exportaciones & $0,0600^{*}$ \\
& $(-0,030)$ \\
Apertura de empresas industriales & $-0,0001$ \\
& $(0,000)$ \\
Cierre de empresas industriales & $-0,0007^{* *}$ \\
& $(0,000)$ \\
Constante & 2,3717 \\
& $(-1,747)$ \\
\hline Test F & $343,27^{* * *}$ \\
R2 $(\mathrm{w})$ & 0,33 \\
\hline Observaciones: 378. & \\
Sig. $* p<0,05 ; * *<0,01 ; * * * p<0,001$ & \\
Efectos fijos por provincia, dummies por año & \\
Variable dependiente: Índice de diversidad productiva \\
Variables rezagadas un período. Errores estándares robustos entre paréntesis. \\
\hline \hline
\end{tabular}

Fuente: elaboración propia en base BADE.

\section{Análisis de robustez}

Con el propósito de contrastar la robustez de los resultados, se probaron diversas especificaciones del modelo que se describen a continuación. En primer lugar, dado que no existen índices de precios para todas las provincias, como alternativa al IPI de Argentina, se toma el índice de precios implícitos (IPI) de Buenos Aires para deflactar los datos PBG (tabla 8). Se observa que los resultados se mantiene, tanto como medida de desarrollo (PBG per cápita - modelo 3) como de proxy de tamaño (PBG- modelo 4). 
Tabla 8 | Factores asociados a la diversidad de la industria regional (Modelo 3 y 4)

\begin{tabular}{lcc}
\multicolumn{1}{c}{ Factores } & Modelo 3 & Modelo 4 \\
\hline \hline PBG (deflactado en base al IPI de & $87,6756^{*}$ & \\
Buenos Aires) per cápita & $(-32,6031)$ & $0,0001^{*}$ \\
PBG (deflactado en base al IPI de & $(0,000)$ \\
Buenos Aires) & $1,0064^{* * *}$ & $0,839^{* * *}$ \\
Densidad & $(-0,1425)$ & $(-0,118)$ \\
& 0,2134 & 0,224 \\
Gasto en I\&D & $(-0,151)$ & $(-0,181)$ \\
& $0,0549^{*}$ & $0,061^{*}$ \\
Participación en las exportaciones & $(-0,0213)$ & $(-0,030)$ \\
& 0,0000 & 0,000 \\
Apertura de empresas industriales & $(-0,0002)$ & $(0,000)$ \\
& $-0,0008^{*}$ & $-0,001^{* *}$ \\
Cierre de empresas industriales & $(-0,0003)$ & $(0,000)$ \\
& 1,9886 & 2,375 \\
Constante & $(-1,5798)$ & $(-1,746)$ \\
\hline Test F & $58,70^{* * *}$ & $354,07^{* * *}$ \\
R2 $(w)$ & 0,38 & 0,33 \\
\hline Observaciones: 378. & & \\
Sig. $* p<0,05 ; * * p<0,01 ; * * p<0,001$ & & \\
Efectos fijos por provincia, dummies por año & & \\
Variable dependiente: Indice de diversidad productiva & \\
Variables rezagadas un período. Errores estándares robustos entre paréntesis. & \\
\hline \hline
\end{tabular}

Fuente: elaboración propia en base BADE.

Otra prueba de robustez consiste en estimar regresiones excluyendo las provincias cuya actividad principal concentra más del $50 \%$ del empleo industrial regional (Tucumán, La Pampa, Entre Ríos, Salta, Río Negro, Mendoza, Jujuy y Tierra del Fuego). Es posible que en dichas regiones la evolución del índice se relacione con factores que afectan la evolución de la rama principal y no con aquellos que afectan la diversidad. Al igual que en la estimación base, la mayoría de las variables explicativas continúan siendo estadísticamente significativas y presentan el signo esperado (tabla 9). Se presentan los resultados tomando el PBGpc constante (deflactado por el IPI de Argentina (modelo 5) y por el de Buenos Aires (modelo 6)). 
Tabla 9 | Excluyendo las provincias con empleo concentrado (Modelo 5 y 6 )

\begin{tabular}{|c|c|c|}
\hline Factores & Modelo 5 & Modelo 6 \\
\hline PBG (deflactado en base al IPI de & $101,409^{* *}$ & \\
\hline Argentina) per cápita & $-29,3258$ & \\
\hline PBG (deflactado en base al IPI de & & $98,9882^{* *}$ \\
\hline Buenos Aires) per cápita & & $-30,6885$ \\
\hline \multirow{2}{*}{ Densidad } & $4,6024^{* *}$ & $4,5532^{* *}$ \\
\hline & $-1,4499$ & $-1,5046$ \\
\hline \multirow{2}{*}{ Gasto en I\&D } & $0,4071^{*}$ & $0,4008^{*}$ \\
\hline & $-0,1698$ & $-0,1712$ \\
\hline \multirow{2}{*}{ Participación en las exportaciones } & $0,0399^{*}$ & $0,0407^{*}$ \\
\hline & $-0,0208$ & $-0,0206$ \\
\hline \multirow{2}{*}{ Apertura de empresas industriales } & 0,0001 & 0,0001 \\
\hline & $-0,0002$ & $-0,0002$ \\
\hline \multirow{2}{*}{ Cierre de empresas industriales } & $-0,0007^{*}$ & $-0,0007^{*}$ \\
\hline & $-0,0003$ & $-0,0003$ \\
\hline \multirow{2}{*}{ Constante } & 0,1987 & 0,2928 \\
\hline & $-1,9722$ & $-1,9928$ \\
\hline Test F & $6,96^{* * *}$ & $9,28^{* * *}$ \\
\hline $\mathrm{R} 2(\mathrm{w})$ & 0,42 & 0,42 \\
\hline
\end{tabular}

Observaciones: 256 .

Sig. $* p<0,05 ; * * p<0,01 ; * * * p<0,001$

Efectos fijos por provincia, dummies por año

Variable dependiente: Índice de diversidad productiva

Variables rezagadas un período. Errores estándares robustos entre paréntesis.

Fuente: elaboración propia en base BADE.

Finalmente, a fines de controlar la posible existencia de un path dependence en el proceso de diversidad, se incluyó la variable dependiente rezagada un período como variable independiente y se modeló a partir del estimador Arellano- Bond para paneles dinámicos (tabla 9). No obstante, la especificación del modelo dinámico (GMM) es globalmente significativa, no así las variables incorporadas, lo cual es evidencia de multicolinealidad entre las variables mencionadas ${ }^{8}$. En algunos trabajos se incluyen variables instrumentales para tratar este problema, no obstante se trata de paneles de mayor dimensión (temporal o en cantidad de individuos) y cuentan con información que no está disponible a nivel provincial en Argentina ${ }^{9}$.

\footnotetext{
${ }^{8}$ En el Anexo IV se incluye la matriz de correlación.

${ }^{9}$ Por ejemplo, He (2009) utiliza datos de 20035 firmas; Elhiraika et al. (2014) trabajan con información de 53 países; Cadot et al. (2011) utilizan datos de exportaciones de 79 países.
} 
Tabla 9 | Factores asociados a la diversidad de la industria regional (Modelo 7)

\begin{tabular}{|c|c|}
\hline Factores & Modelo 7 \\
\hline Índice de diversidad (rezagado un período) & $\begin{array}{c}-0,5481 \\
(-0,7465)\end{array}$ \\
\hline PBG per cápita & $\begin{array}{c}-17,06 \\
(-441,1592)\end{array}$ \\
\hline Densidad & $\begin{array}{c}-13,8913 \\
(-14,1142)\end{array}$ \\
\hline Gasto en I\&D & $\begin{array}{c}0,4415 \\
(-0,9466)\end{array}$ \\
\hline Participación en las exportaciones & $\begin{array}{c}-0,0098 \\
(-0,0468)\end{array}$ \\
\hline Apertura de empresas industriales & $\begin{array}{c}0,0003 \\
(-0,0004)\end{array}$ \\
\hline Cierre de empresas industriales & $\begin{array}{c}-0,0004 \\
(-0,0005)\end{array}$ \\
\hline Constante & $\begin{array}{c}7,3263 \\
(-4,5333)\end{array}$ \\
\hline Test de Wald & $1904,06^{* * *}$ \\
\hline $\begin{array}{l}\text { Observaciones: } 378 \text {. } \\
\text { Sig. } * p<0,05 ; * * p<0,01 ; * * * p<0,001 \\
\text { Efectos fijos por provincia, dummies por añ } \\
\text { Variable dependiente: Índice de diversidad p } \\
\text { Variables rezagadas un período. Errores está }\end{array}$ & ustos entre paréntesis. \\
\hline
\end{tabular}

Fuente: elaboración propia en base BADE.

\section{Reflexiones finales}

La diversidad productiva es señalada en la literatura como un instrumento clave en el diseño de políticas industriales de desarrollo regional. Una estructura productiva más diversa se asocia a un ambiente propicio a nuevas inversiones, mayor innovación, transmisión de conocimientos, entre otros. En esta línea, esta tesis contribuye empíricamente al estudio del fenómeno, mediante un abordaje regional con datos actualizados para todas las provincias argentinas. En particular, se analiza la diversidad industrial tanto en términos estáticos como dinámicos: por un lado, se describe y cuantifica el grado de diversidad productiva regional en Argentina y su evolución a lo largo del período 1996-2012, y por otro, se examina la relación entre dicho fenómeno y algunos factores económicos regionales asociados.

En términos estáticos, las jurisdicciones más diversas del país son CABA, Buenos Aires, Santa Fe, Córdoba, San Luis y Neuquén. Este resultado puede estar asociado con el mayor grado de urbanismo de estas regiones, en un entorno de mayores capacidades productivas, 
institucionales y organizativas que incentiven la radicación de un gran número de empresas de diversos sectores. En contraposición con este grupo, las provincias menos diversas son las de base agro-alimentaria (Tucumán, La Pampa, Entre Ríos, Salta, Río Negro, Mendoza y Jujuy), puesto que la rama Alimentos y Bebidas concentra más del $50 \%$ del empleo industrial.

En términos dinámicos, la diversidad productiva es un fenómeno pro-cíclico, tanto a nivel nacional como en la mayor parte de las regiones. Las provincias favorecidas con regímenes de promoción industrial (San Luis, Catamarca, San Juan, La Rioja y Tierra del Fuego) constituyen un caso particular donde la diversidad industrial disminuye en forma continua. Esto puede estar asociado al impacto decreciente de dichos regímenes sobre la estructura productiva. Asimismo, los resultados muestran que los aumentos en la diversidad pueden ser producto tanto del crecimiento del empleo en ramas poco tradicionales (Neuquen y Chubut) o de una disminución del empleo en la rama principal (Chaco).

Los resultados de la estimación indican que la diversidad industrial de las provincias se asocia positivamente con el grado de desarrollo, el tamaño de la región y una mayor urbanización. Esto implica una restricción al momento de fomentar la diversificación productiva a través de políticas específicas, dado que estas características estructurales son difíciles de modificar en el corto plazo. La diversidad industrial regional también se relaciona en forma directa con las capacidades territoriales, que podrían ser impulsadas a través de políticas industriales a mediano plazo.

Por otra parte, la diversidad industrial se asocia negativamente a la salida de empresas. Este resultado podría relacionarse con el cierre de firmas en sectores menos tradicionales, cuyas economías externas (mercado de trabajo especializado, proveedores, etc.) e instituciones de apoyo no están lo suficientemente consolidadas. Al respecto, políticas de retención de empresas en sectores menos tradicionales podrían ser más efectivas para aumentar la diversidad que aquellas orientadas al ingreso de nuevos emprendimientos en dichos sectores.

Algunas dimensiones sobre diversidad industrial que no han sido abordadas en la presente tesis dan lugar a potenciales investigaciones en la temática. En primer lugar, un 
mayor nivel de desagregación de los datos (3 o 4 dígitos CIIU), así como el estudio de los sectores primario, comercial y de servicios permitirían comprender con mayor profundidad el fenómeno. Asimismo, sería interesante identificar si la diversidad tiene lugar en industrias relacionadas o no, puesto que la literatura internacional indica que estos dos tipos de diversidad son impulsados por diferentes fuentes y tienen distintos efectos. En la misma línea, la disponibilidad de datos de demografía empresarial con mayor apertura sectorial permitiría identificar en qué ramas de actividad (tradicionales o no) se producen efectivamente las entradas y salidas de empresas. Por último, se plantea la posibilidad de avanzar desde el punto de vista metodológico, a partir del cálculo de nuevos indicadores de diversidad ponderados por el peso relativo del empleo industrial en la región. 


\section{Referencias}

Al-Marhubi, F. (2000). Export diversification and growth: an empirical investigation. Applied Economics Letters, 7 (9), 559-562.

Aláez Aller, R; Longás García, J; Ullibarri Arce, M; (2001). La relación entre efectos externos y aglomeración: una aproximación a su estudio a partir de la evidencia empírica disponible. Revista de Estudios Regionales, septiembre-diciembre, 151-167.

Aw, B., y Batra, G. (1998). Firm size and the pattern of diversification.International Journal of Industrial Organization, 16(3), 313-331.

Baltagi, B. (2008). Econometric analysis of panel data. John Wiley \& Sons.

Beaudry, C., y Schiffauerova, A. (2009). Who's right, Marshall or Jacobs? The localization versus urbanization debate. Research policy, 38(2), 318-337.

Bebczuk, R. y Berrettoni, D. (2006). Explaining Export Diversification: An Empirical Analysis, Documentos de Trabajo. Universidad Nacional de La Plata.

Beckstead, D., y Brown, W. (2007). From Labrador City to Toronto: The Industrial Diversity of Canadian Cities, 1992 to 2002. Insights on the Canadian Economy.

Bishop, P., yGripaios, P. (2007). Explaining spatial patterns of industrial diversity: an analysis of sub-regions in Great Britain. Urban Studies, 44(9), 1739-1757.

Boschma R. (2004). Competitiveness of regions from an evolutionary perspective, Regional Studies 38(9): 1001-1014.

Boschma, R., y Iammarino, S. (2007). Related variety and regional growth in Italy. Science and Technology Policy Research, (62), 1-24.

Boschma, R., Minondo, A., y Navarro, M. (2013). The emergence of new industries at the regional level in Spain: a proximity approach based on product relatedness. Economic Geography, 89(1), 29-51.

Boschma, R., y Capone, G. (2015). Institutions and diversification: Related versus unrelated diversification in a varieties of capitalism framework. Research Policy, 44(10), 1902-1914.

Cadot, O., Carrère, C., y Strauss-Kahn, V. (2011). Export diversification: What's behind the hump?. Review of Economics and Statistics, 93(2), 590-605.

Capello, R. (2006). La Economía Regional tras cincuenta años: Desarrollos teóricos recientes y desafíos futuros/Regional Economics in its Fifties: Recent Theoretical Directions and Future Challenges. Investigaciones regionales, (9), 169.

Castellacci, F. (2007). Technological regimes and sectoral differences in productivity growth. Industrial and corporate change, 16(6), 1105-1145.

Castillo, V., Ferlan, E., Mazorra, X., Schleser, D., Stumpo, G., Yoguel, G., y Rojo Brizuela, S. (2004). Observatorio de empleo y dinámica empresarial en Argentina. CEPAL.

Crépon, B., Duguet, E. y Mairesse, J. (1998). Research, innovation and productivity: An econometric analysis at the firm level. Economics of Innovation and New Technology, $7(2), 115-158$.

De Benedictis, L., Gallegati, M., y Tamberi, M. (2009). Overall trade specialization and economic development: countries diversify. Review of World Economics,145(1), 37-55. 
Dewhurst, J. L., y McCann, P. (2002). A comparison of measures of industrial specialization for travel-to-work areas in Great Britain, 1981-1997. Regional Studies, 36(5), 541-551.

Donato, V. (2007). Políticas Públicas y localización industrial en Argentina. Revista OIDLES, 1(1).

Dumais, G., Ellison, G. Glaeser E., (2002): Geographic Concentration As A Dynamic Process. The Review of Economics and Statistics, 84, 193-204.

Duranton, G. y Puga, D. (2000). Diversity and specialisation in cities: why, where and when does it matter?. Urban studies, 37 (3), 533-555.

Elhiraika, A., y MBATE, M. (2014). Assessing the determinants of export diversification in Africa. Applied Econometrics and International Development, 14(1), 147-160.

Essletzbichler, J., y Rigby, D. L. (2007). Exploring evolutionary economic geographies. Journal of Economic Geography.

Filipescu, D. A., Prashantham, S., Rialp, A., y Rialp, J. (2013). Technological innovation and exports: Unpacking their reciprocal causality. Journal of International Marketing, 21(1), 23-38.

Feldman, M. P., y Audretsch, D. B. (1999). Innovation in cities:: Science-based diversity, specialization and localized competition. European economic review,43(2), 409-429.

Frenken, K., van Oort, F. G., Verburg, T., y Boschma, R. A. (2004). Variety and regional economic growth in the Netherlands (0502). Utrecht University, Section of Economic Geography.

Frenken, K., Van Oort, F., y Verburg, T. (2007). Related variety, unrelated variety and regional economic growth. Regional studies, 41(5), 685-697.

Fujita, M., y Krugman, P. (2004). La nueva geografía económica: pasado, presente y futuro/The new economic geography: past, present and future. Investigaciones Regionales, (4), 177.

Gatto, F. (2007). Crecimiento económico y desigualdades territoriales: algunos límites estructurales para lograr una mayor equidad. Crisis, recuperación y nuevos dilemas, 307.

Ghosh, A. y Ostry, J. (1994). Export instability and the external balance in developing countries, IMF Working Paper 94/8, Washington DC: International Monetary Fund.

Glaeser, E. , Kallal, H., Scheinkman, J., y Shleifer, A.(1992). Growth in Cities. Journal of Political Economy, 100(6), 1126-1152.

Guesnier, B. (1994). Regional variations in new firm formation in France. Regional Studies, 28 (4), 347-358.

Haddad, M., Lim, J., y Saborowski, C. (2010). Trade Openness Reduces Growth Volatility When Countries Are Well Diversified. WPS 522, World Bank Policy research Working Paper, Washington: The World Bank.

Hammouda, H., Karingi, S., Njuguna, A., y Sadni Jallab, M. (2006). Diversification: towards a new paradigm for Africa's development (13359). University Library of Munich, Alemania.

Hausman, J. A. (1978). Specification tests in econometrics. Econometrica: Journal of the Econometric Society, 1251-1271. 
He, X. (2009). Corporate Diversification and Firm Value: Evidence from Post 1997 Data. International Review of Finance, 9(4), 359-385.

Herzer, D., y Nowak-Lehnmann, D. (2006). What does export diversification do for growth? An econometric analysis. Applied economics, 38 (15), 1825-1838.

Hesse, H. (2009). Export Diversification and Economic Growth, en R.S. Newfarmer, W. Shaw and P. Walkenhorst (eds), Breaking into New Markets: Emerging Lessons for Export Diversification, Washington, DC: The World Bank.

Hidalgo, C. A., Klinger, B., Barabási, A. L., y Hausmann, R. (2007) The product space conditions the development of nations. Science, 317(5837), 482-487.

Hoover, E. M. (1936). The measurement of industrial localization. The Review of Economic Statistics, 162-171.

Imbs, J., y Wacziarg, R. (2003). Stages of diversification. American Economic Review, $63-86$.

Kamien, M. I., y Schwartz, N. L. (1975). Market structure and innovation: A survey.Journal of economic literature, 13(1), 1-37.

Klinger, B., y Lederman, D. (2004). Discovery and development: an empirical exploration of "new" products (3450). World Bank Publications.

Kosacoff, B., y Ramos, A. (1999). El debate sobre política industrial. Revista de la CEPAL.

Krugman, P. (1991). Increasing Returns and Economic Geography. The Journal of Political Economy, 99(3), 483-499.

Krugman, P. (1995). Development, Geography and Economic Theory. MIT Press.

Krugman, P. (1998). What's new about the new economic geography?. Oxford review of economic policy, 14(2), 7-17.

Krugman, P. (1999). The Role of Geography in Development. International Regional Science Review, 22(2): 142-161.

Lavarello, P. J., y Sarabia, M. (2015). La política industrial en la Argentina durante la década de 2000.CEPAL.

Lawson, C. (1999). Towards a competence theory of the region. Cambridge Journal of Economics, 23(2), 151-166.

Mack, E., Grubesic, T. y Kessler, E. (2007). Indices of industrial diversity and regional economic composition. Growth and Change, 38(3), 474-509.

Marra, A., Carlei, V., y Paglialunga, A. (2011). Economic Diversity and Industry Patterns in Italian Provinces: An Empirical Analysis. L'industria, 32(3), 419-434.

Martin, R., y Sunley, P. (2006). Path dependence and regional economic evolution. Journal of economic geography, 6(4), 395-437.

Mazorra, X. y Beccaria, A. (2007). "Especialización productiva y empleo en Áreas Económicas Locales". En: Novick, M.; Palomino, H. (Coord.). 2007. Estructura productiva y empleo. Un enfoque transversal,Buenos Aires, Ministerio de Trabajo, Empleo y Seguridad Social. 
McCann, P., y van Oort, F. (2009). Theories of agglomeration and regional economic growth: a historical review. Handbook of regional growth and development theories, 19-32.

Ministerio de Industria (2011)Plan Estratégico Industrial 2020. Ministerio de Industria, Presidencia de la Nación, Argentina.

Monastiriotis, V. (2000). City size and production diversity: patterns of specialisation and diversity in the US cities, 1969-1997. 40th European Regional Science Congress, Barcelona.

Moncayo Jiménez, E. (2001). Evolución de los paradigmas y modelos interpretativos del desarrollo territorial. CEPAL.

Montgomery, C. A. (1994). Corporate diversification. The Journal of Economic Perspectives, 8(3), 163-178.

Nachum, L. (2004). Geographic and Industrial Diversification of Developing Country Firms. Journal of Management Studies, 41(2), 273-294.

Neffke, F., Henning, M., y Boschma, R. (2011). How do regions diversify over time? Industry relatedness and the development of new growth paths in regions. Economic Geography, 87(3), 237-265.

Neffke, F., Hartog, M., Boschma, R., y Henning, M. (2014). Agents of structural change. The role of firms and entrepreneurs in regional diversification (1410). Utrecht University, Section of Economic Geography.

Noseleit, F. (2011). Market Selection and Regional Diversification-Empirical Regularities from German Panel-Data. European Regional Science Association.

Parteka, A., y Tamberi, M. (2013). Product diversification, relative specialisation and economic development: Import-export analysis. Journal of Macroeconomics,38, 121-135.

Penrose, E. T. (1959). The Theory of the Growth of the Firm. New York: John Wiley.

Porta, F. (2016) La estructura productiva argentina. Problemas vigentes y desafíos pendientes. Voces en el Fenix, 53: 90-97.

Ramanujam, V., y Varadarajan, P. (1989). Research on corporate diversification: A synthesis. Strategic Management Journal, 10(6), 523-551.

Reynolds, P., Storey, D. y Westhead, P. (1994). "Cross-national comparisons of the variation in new firm formation rates", Regional Studies, 28(4): 443-456.

Rodgers, A. (1957). Some aspects of industrial diversification in the United States. Economic Geography, 16-30.

Rojo Brizuela, S., y Rotondo, S. (2006). Especialización industrial y empleo registrado en el Gran Buenos Aires. Informe de la Dirección de Estudios y Estadísticas Laborales de la Subsecretaría de Programación Técnica y Estudios Laborales, en Trabajo, ocupación y empleo, Serie Estudios, Ministerio de Trabajo, Empleo y Seguridad Social.

Schmookler, J. (1966). Invention and economic growth. Cambridge, MA: Harvard University Press.

Teece, D. J. (1980). Economies of scope and the scope of the enterprise. Journal of economic behavior 85 organization, 1(3), 223-247.

Wooldridge, J. (2010). Econometric analysis of cross section and panel data. MIT.

Zalduendo, E. (1973). Las desigualdades económicas entre regiones de Argentina, Buenos Aires. Las desigualdades económicas entre regiones de Argentina. 


\section{Anexo I. Clasificación Industrial Internacional Uniforme}

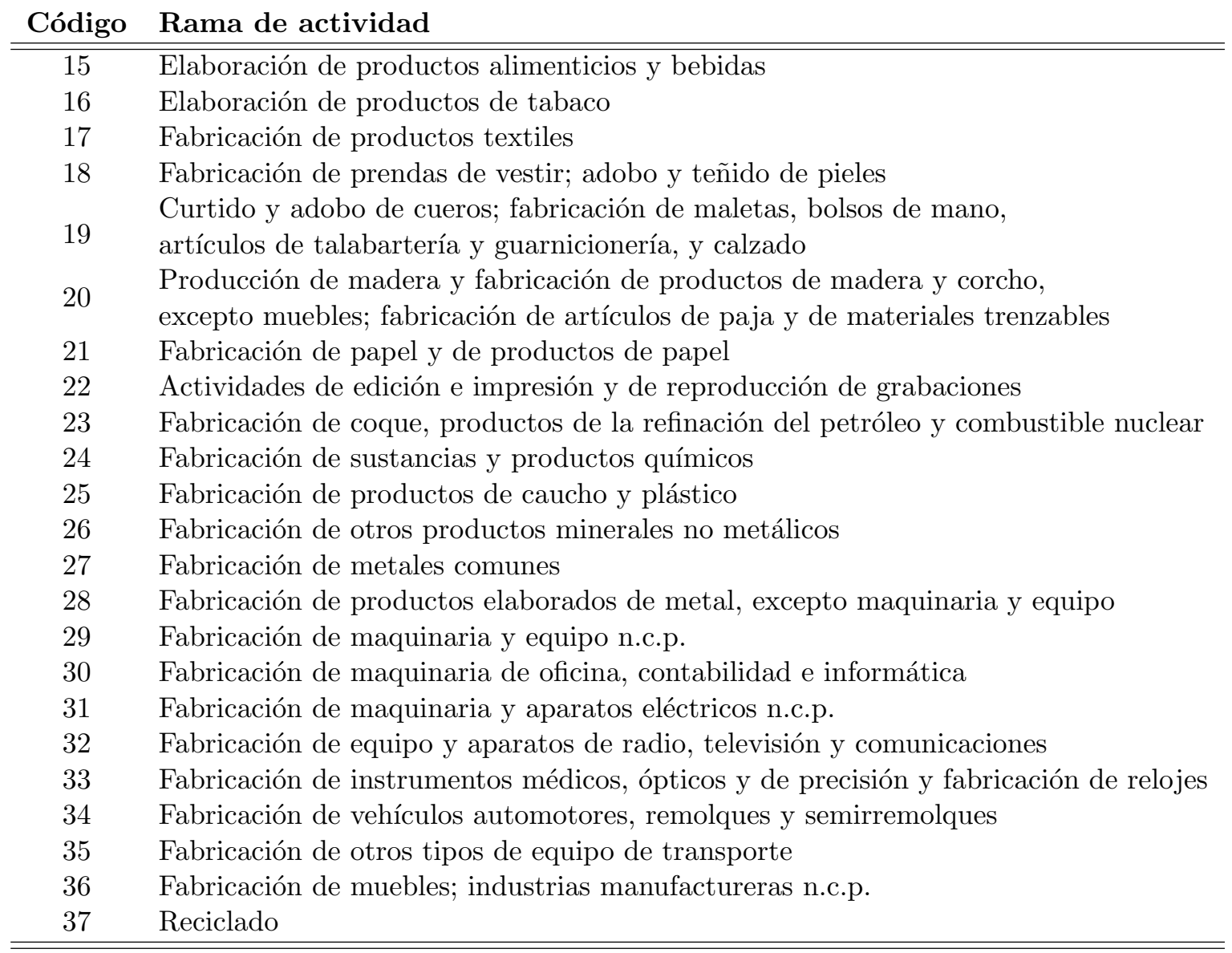

Fuente: elaboración propia en base a CIIU Revisión 3.1. n.c.p: no clasificado previamente 


\section{Anexo II. Evolución del índice de diversidad productiva por provincias}

Figura 4 | Provincias centrales

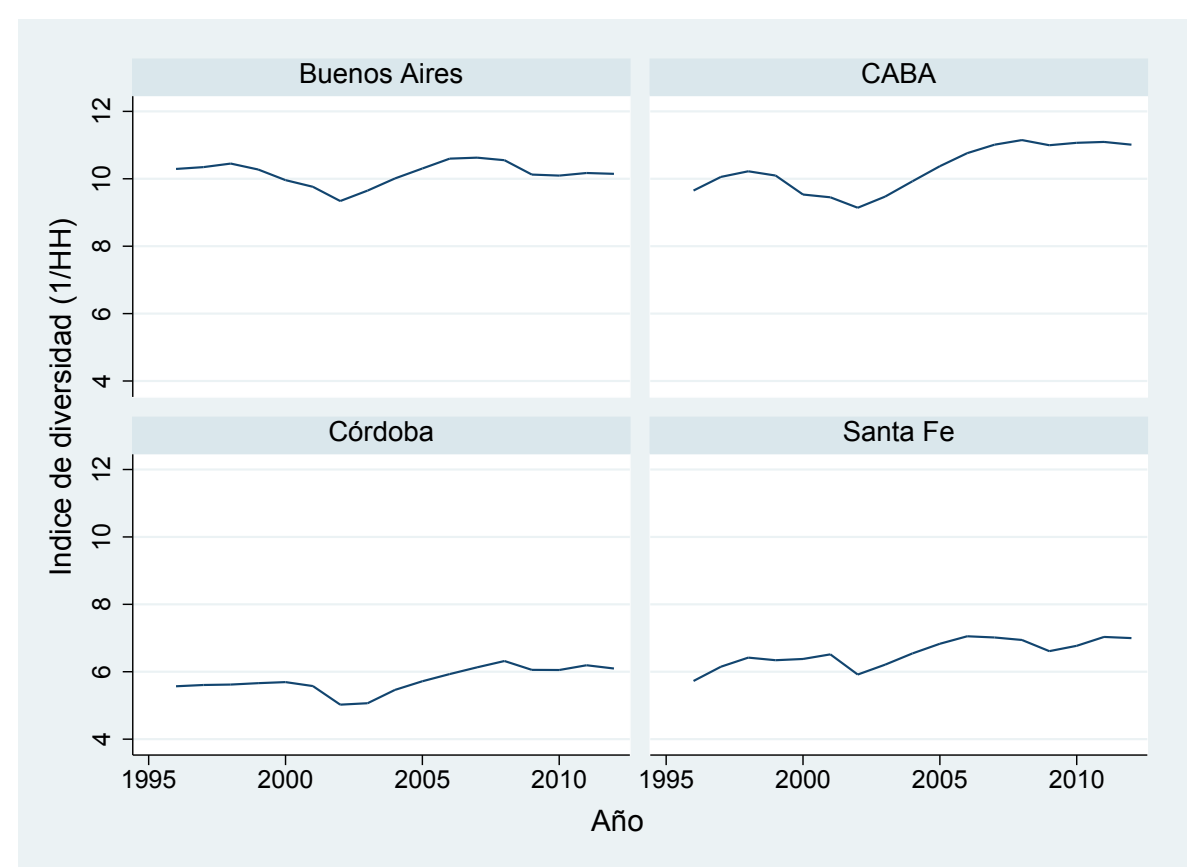

Fuente: elaboración propia en base BADE.

Figura 5 | Provincias con regímenes de promoción industrial

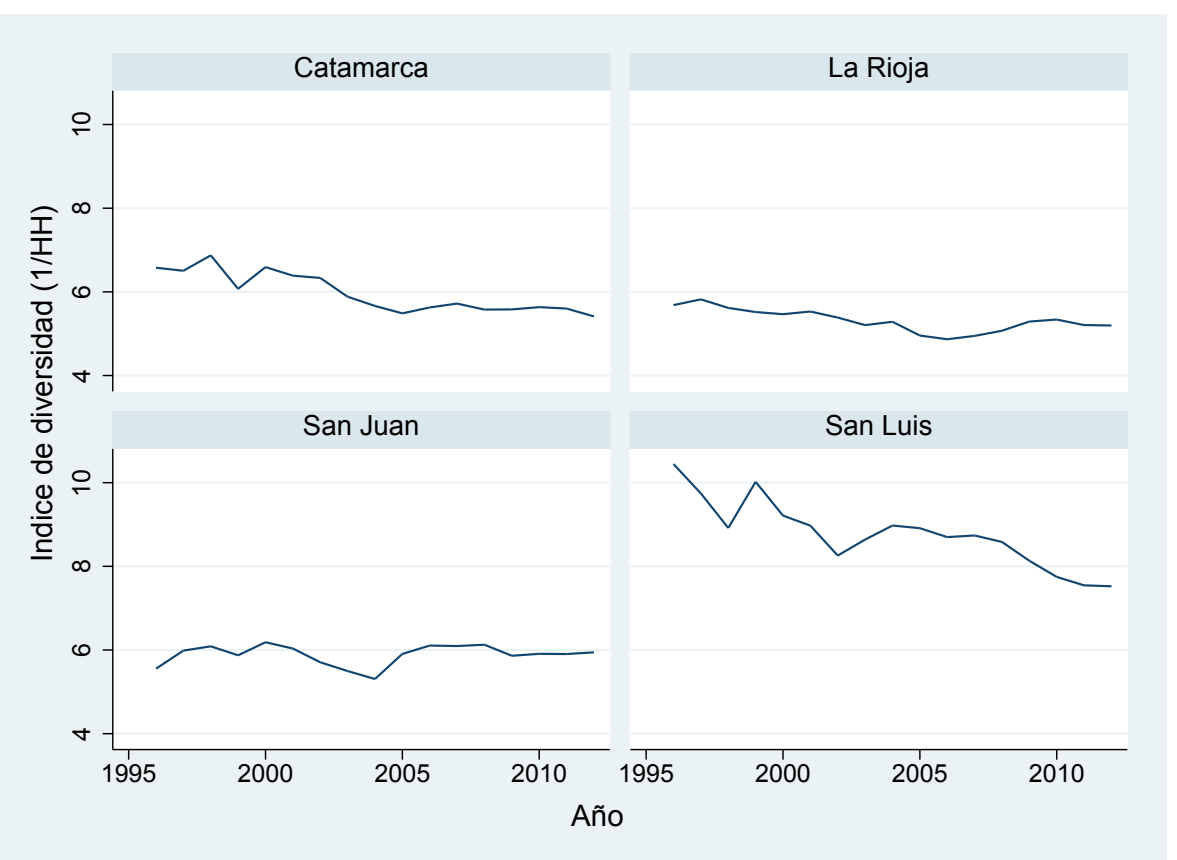

Fuente: elaboración propia en base BADE. 
Figura 6 | Provincias patagónicas

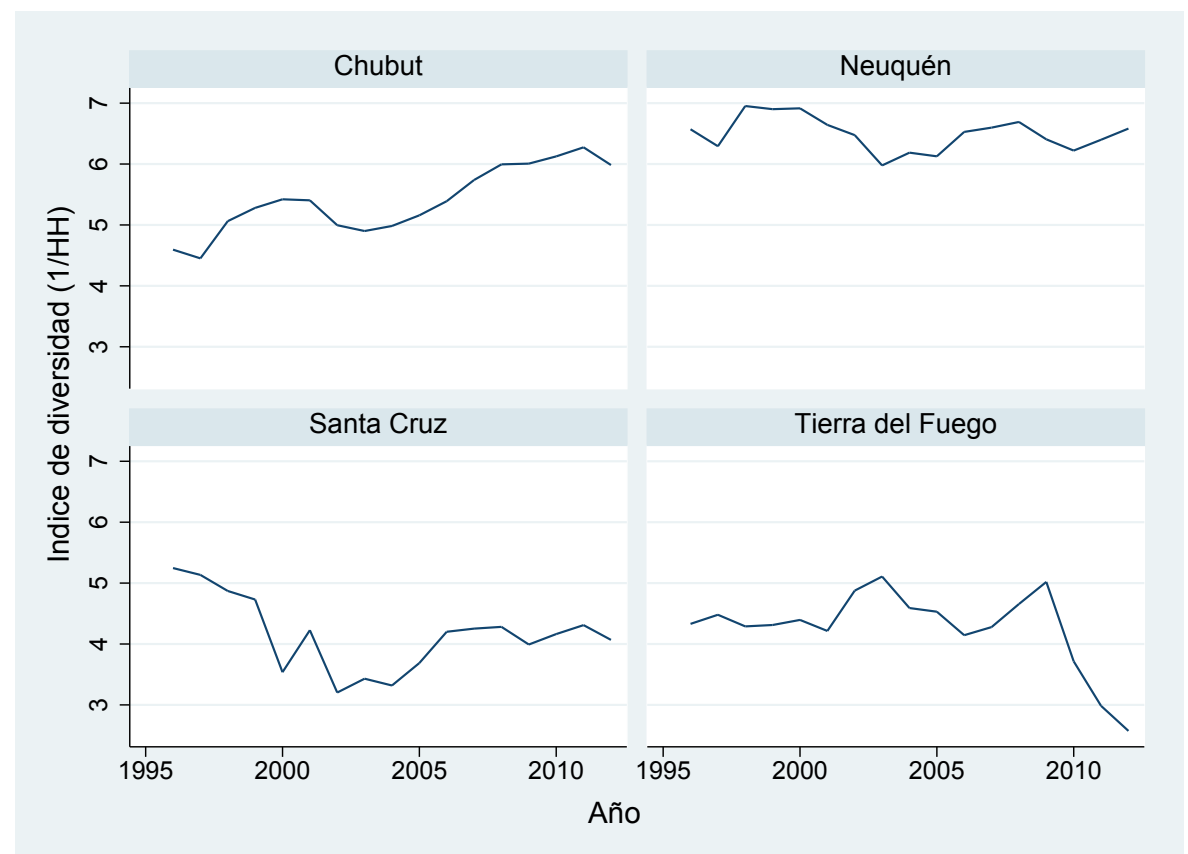

Fuente: elaboración propia en base BADE.

Figura 7 | Provincias del noreste

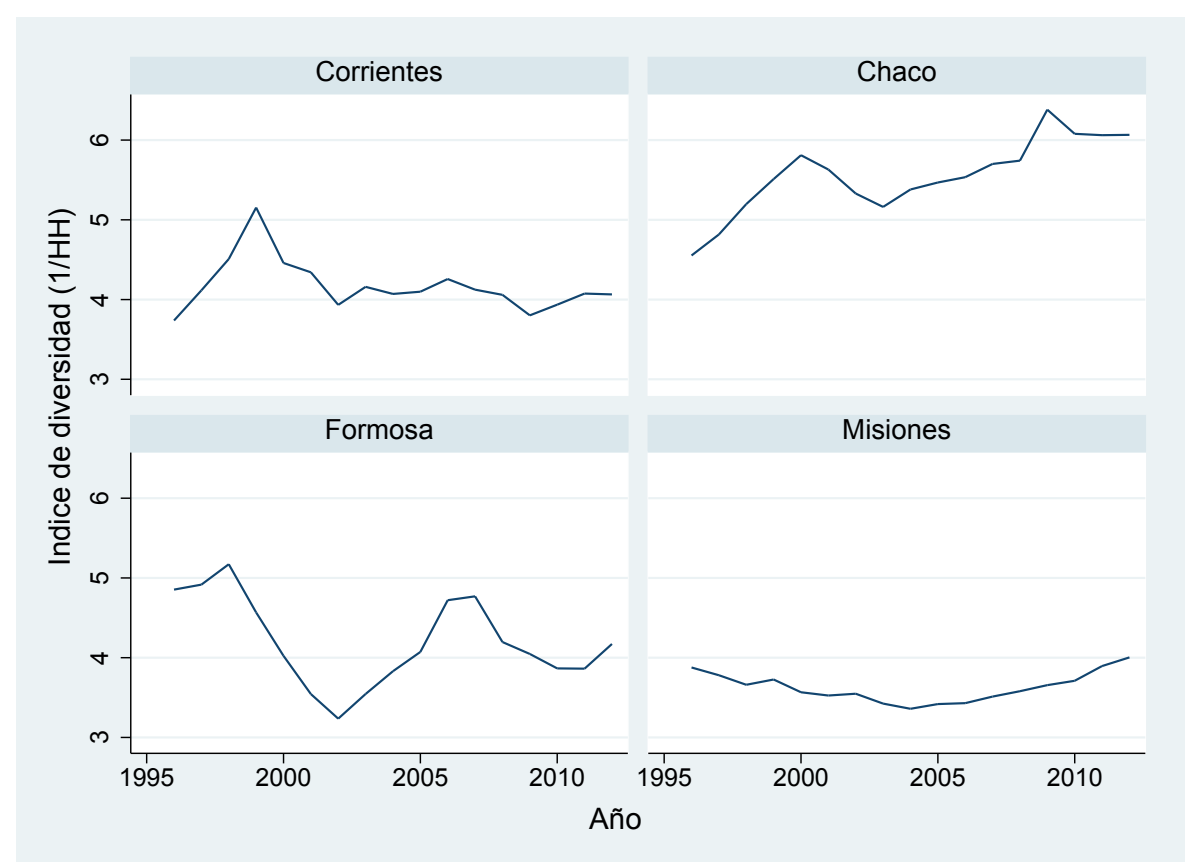

Fuente: elaboración propia en base BADE. 
Figura 8 | Provincias de base agro-industrial

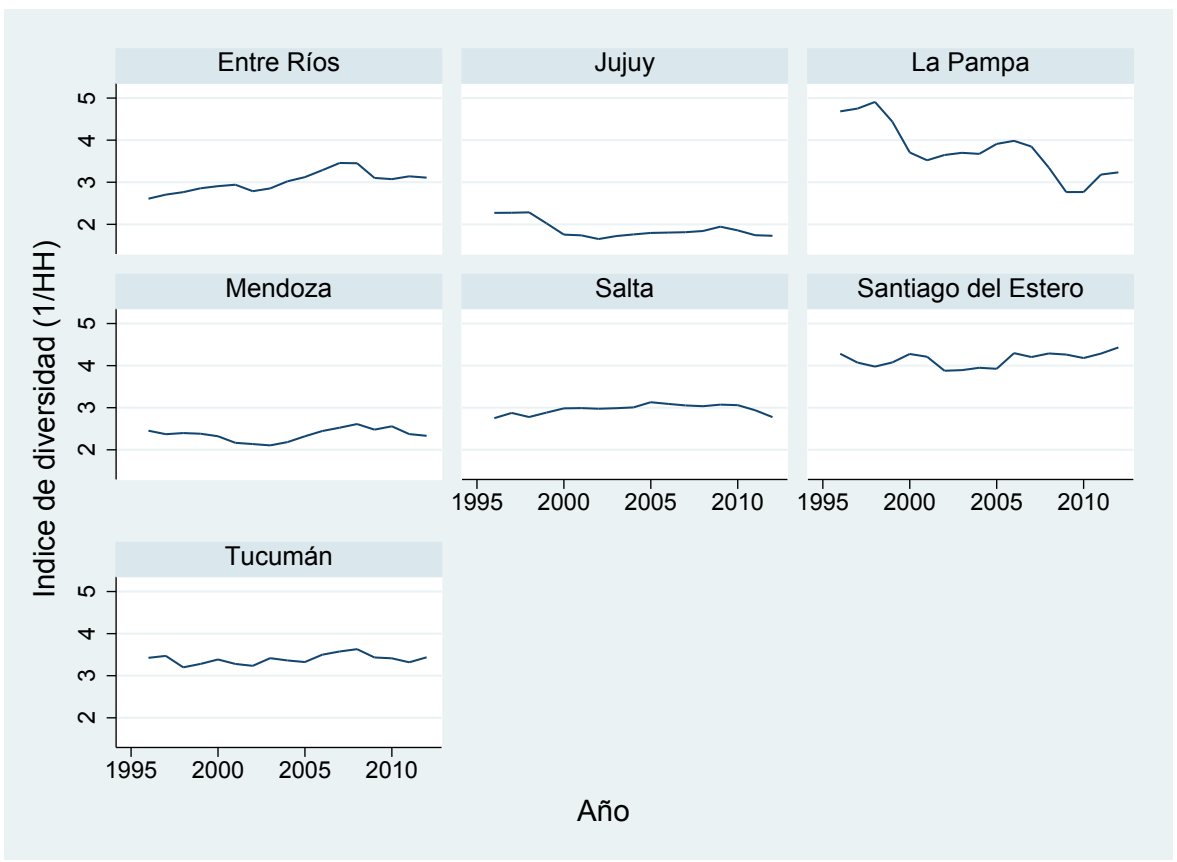

Fuente: elaboración propia en base BADE. 


\section{Anexo III. Evolución del empleo industrial por rama de ac- tividad}

Figura 9 | Tierra del Fuego

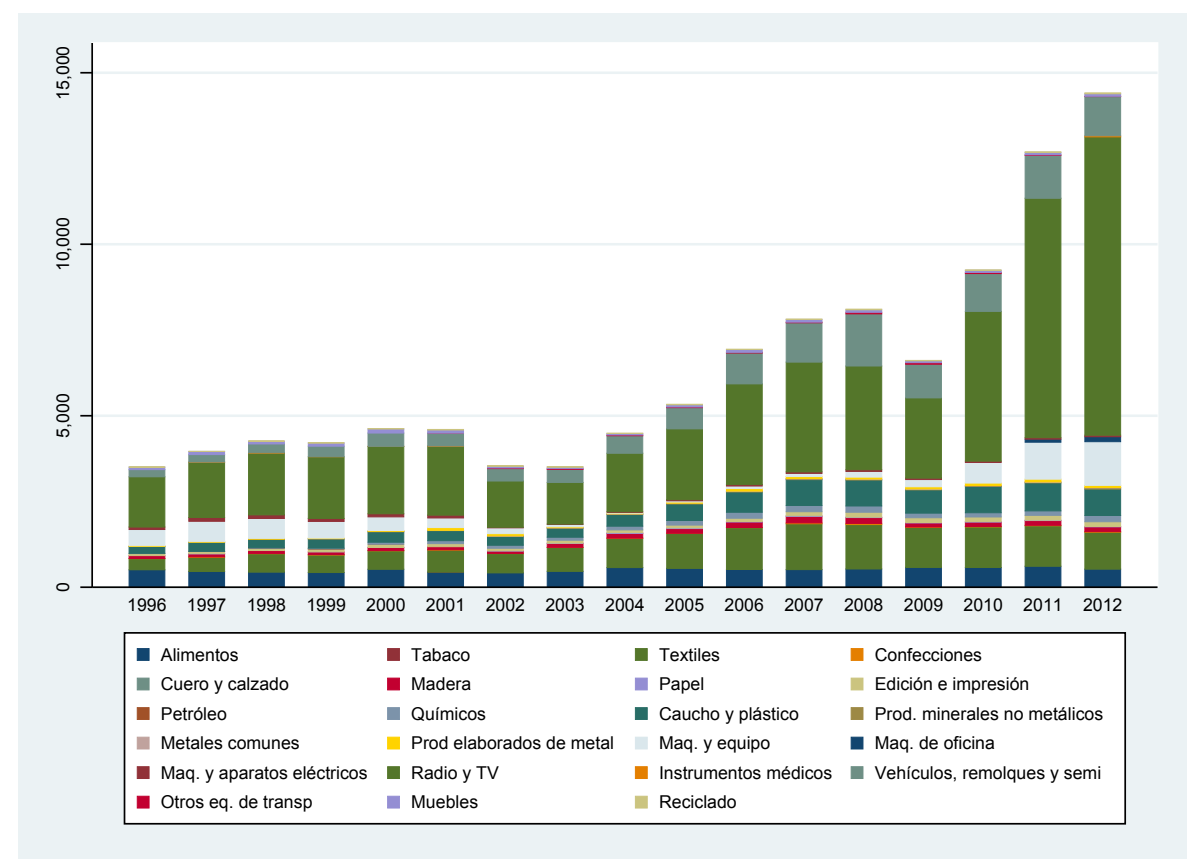

Fuente: elaboración propia en base BADE.

Figura 10 | Neuquén

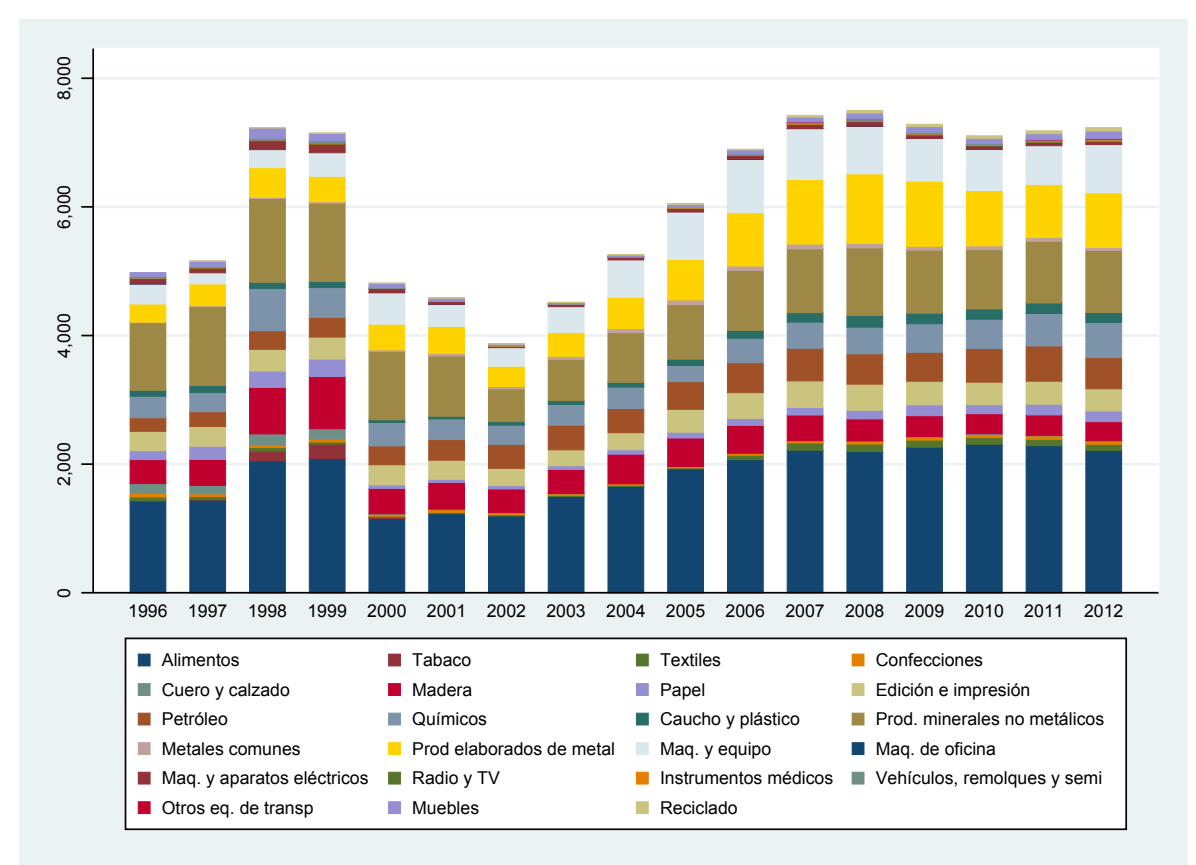

Fuente: elaboración propia en base BADE. 
Figura 11 | Chaco

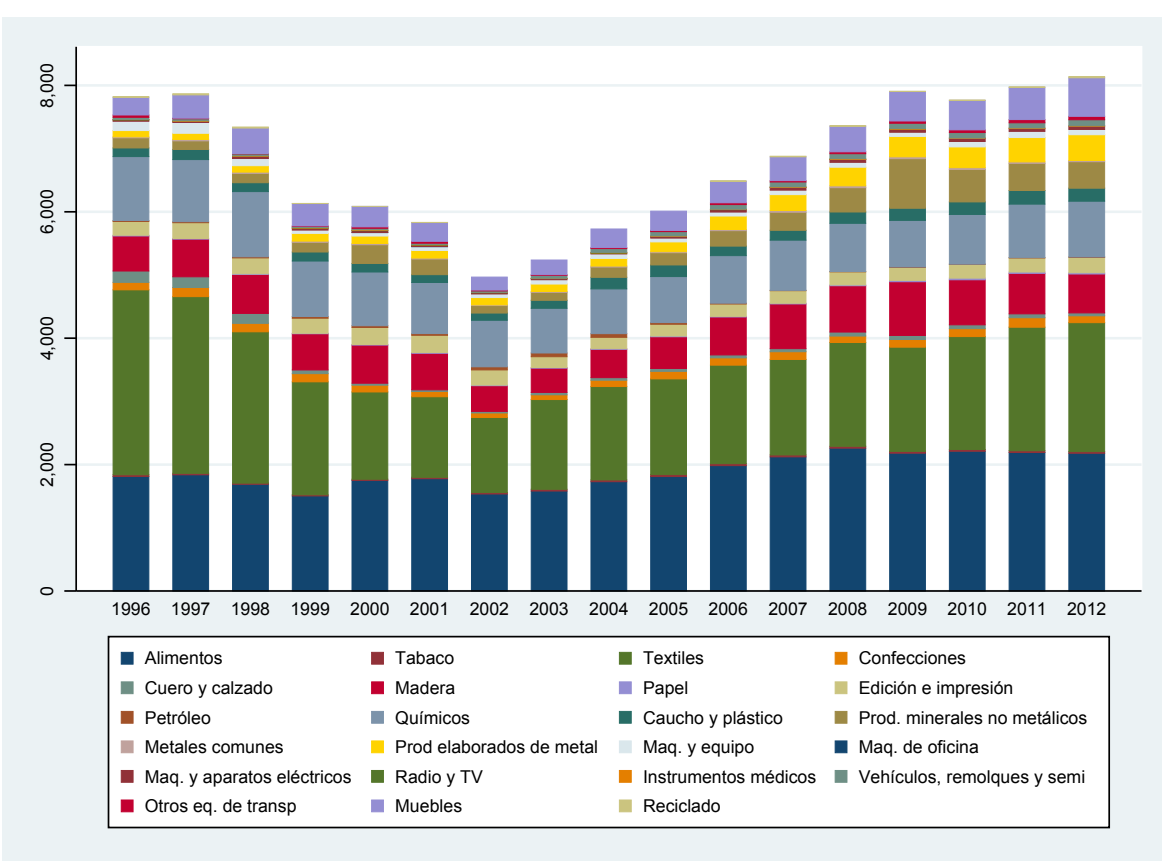

Fuente: elaboración propia en base BADE.

Figura $12 \mid$ Corrientes

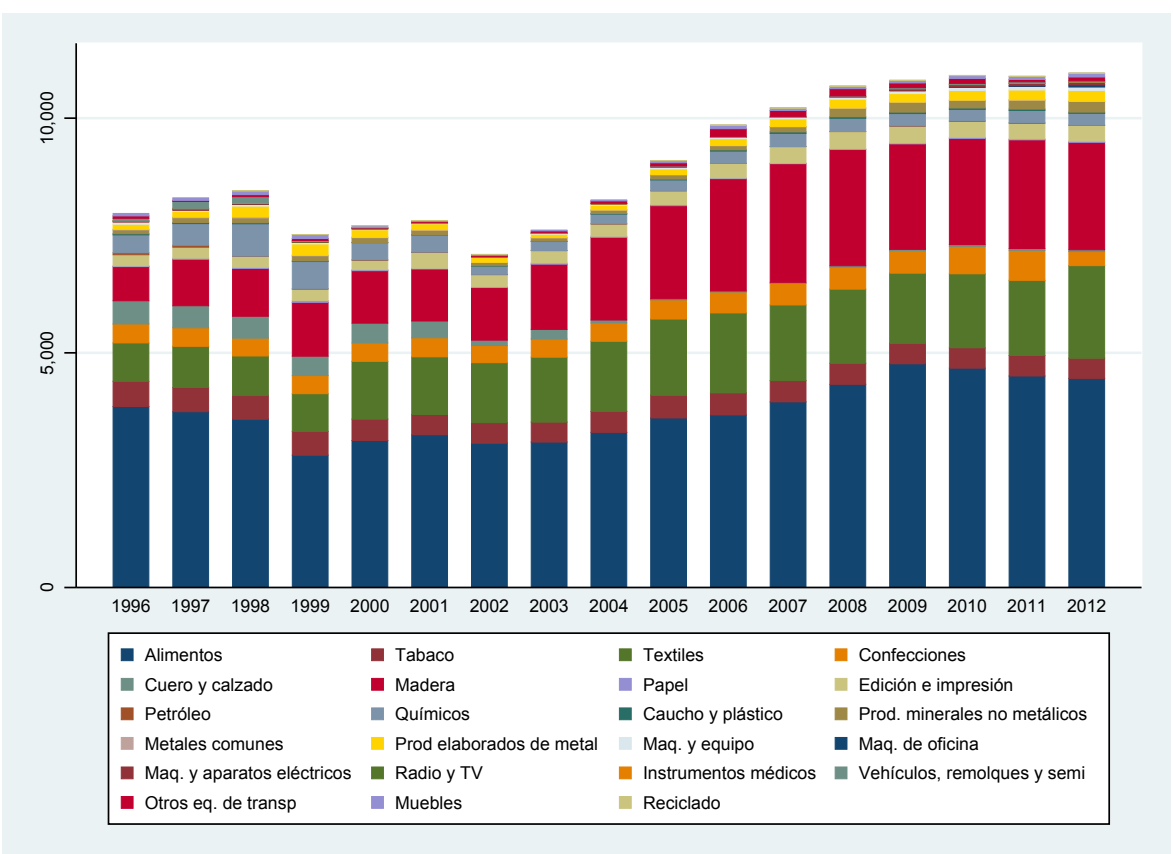

Fuente: elaboración propia en base BADE. 
Figura 13 | Mendoza

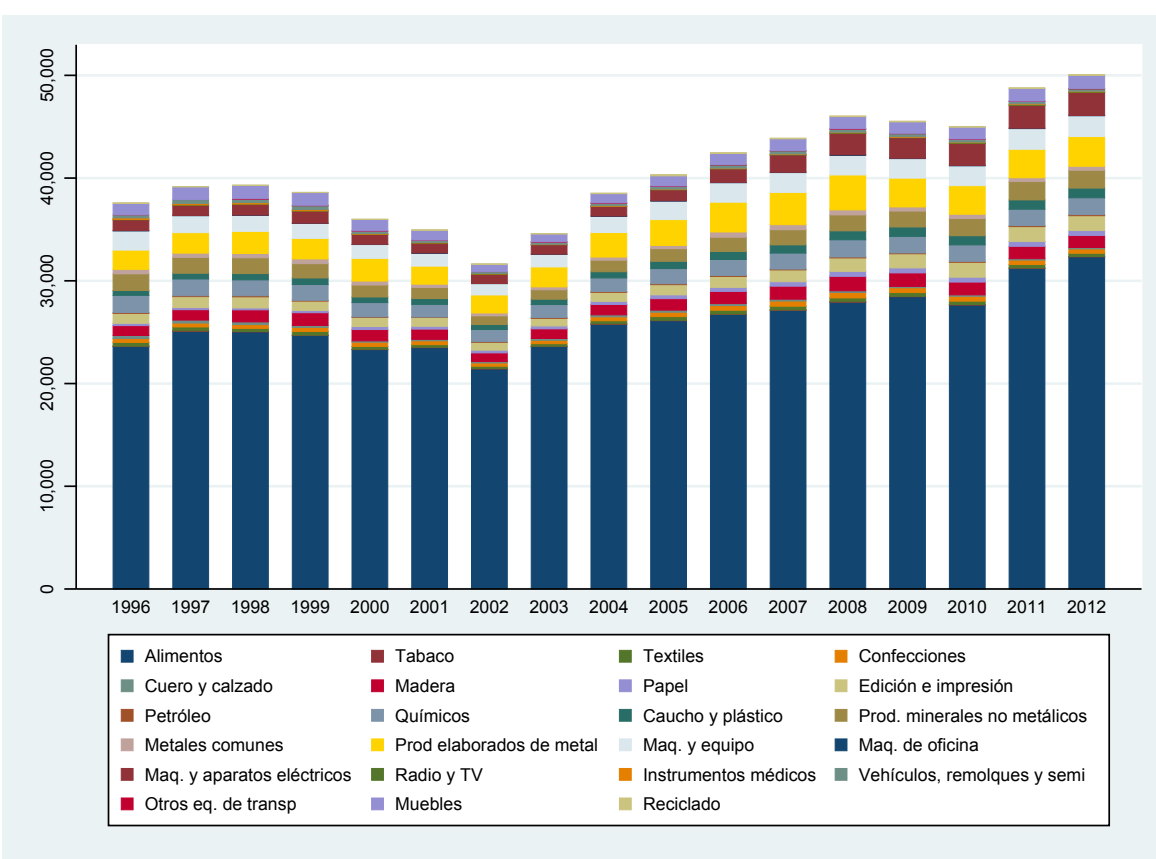

Fuente: elaboración propia en base BADE.

Figura 14 | Entre Ríos

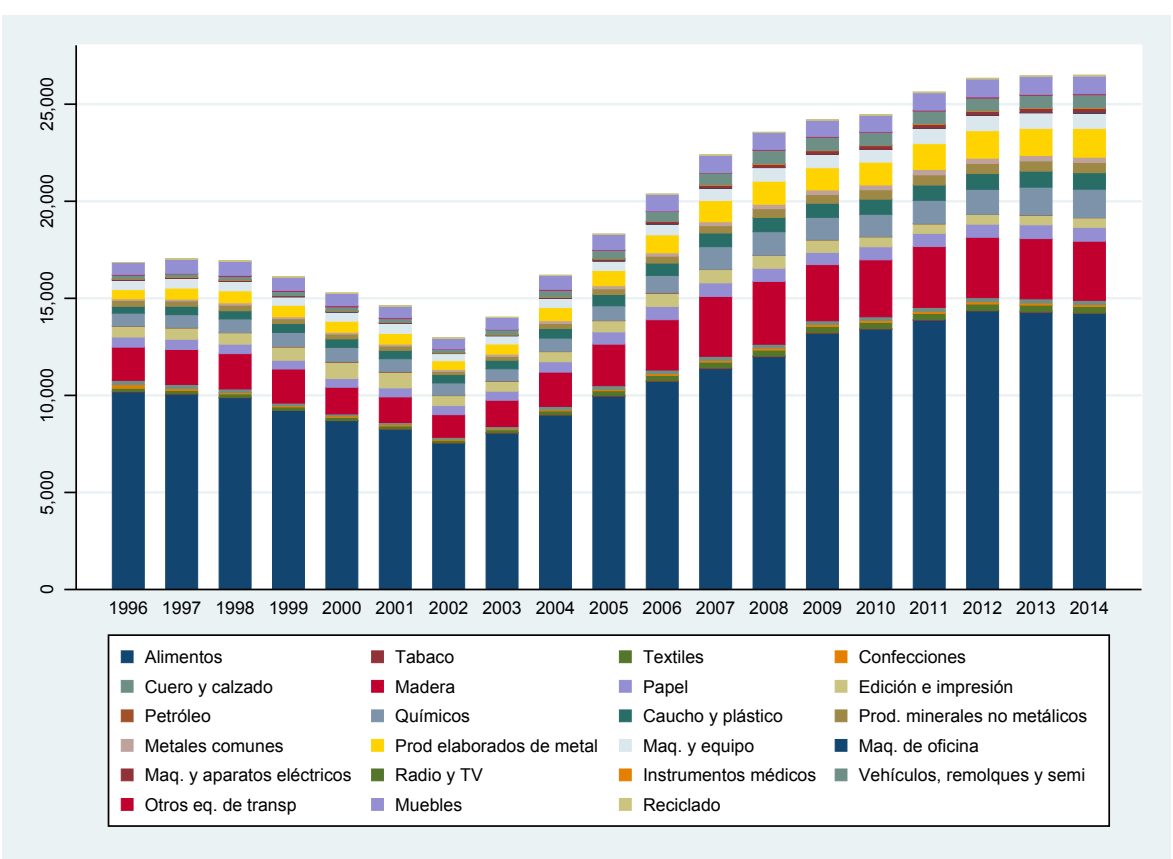

Fuente: elaboración propia en base BADE. 